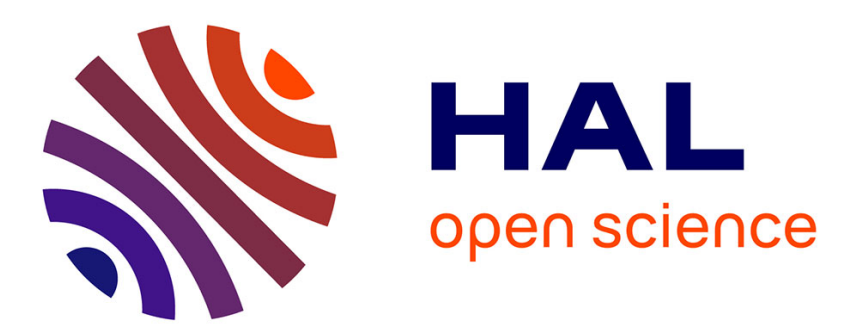

\title{
Computation of energy release rate using material differentiation of elastic BIE for 3-D elastic fracture
}

Marc Bonnet, H. Xiao

\section{To cite this version:}

Marc Bonnet, H. Xiao. Computation of energy release rate using material differentiation of elastic BIE for 3-D elastic fracture. Engineering Analysis with Boundary Elements, 1995, 15, pp.137-150. 10.1016/0955-7997(95)00012-D . hal-00092366

\section{HAL Id: hal-00092366 https://hal.science/hal-00092366}

Submitted on 9 Aug 2008

HAL is a multi-disciplinary open access archive for the deposit and dissemination of scientific research documents, whether they are published or not. The documents may come from teaching and research institutions in France or abroad, or from public or private research centers.
L'archive ouverte pluridisciplinaire HAL, est destinée au dépôt et à la diffusion de documents scientifiques de niveau recherche, publiés ou non, émanant des établissements d'enseignement et de recherche français ou étrangers, des laboratoires publics ou privés. 


\title{
Computation of energy release rate using material differentiation of elastic BIE for 3-D elastic fracture ${ }^{1}$
}

\author{
Marc BonneT ${ }^{2}$, Haihong XIAO \\ Laboratoire de Mécanique des Solides
}

(URA CNRS 317, centre commun Polytechnique - Mines - Ponts et Chaussées)

Ecole Polytechnique, 91128 Palaiseau Cedex, France

bonnet@athena.polytechnique.fr, (1)-69333026 (fax)

${ }^{1}$ Eng. Anal with Bound. Elem., 15:137-150 (1995)

${ }^{2}$ to whom correspondence should be addressed 


\begin{abstract}
This paper deals with a novel approach, based on material differentiation of elastic BIE formulations, for the numerical computation of the energy release rate $G$ along a crack front in 3-D elastic fracture problems. It is based upon the definition of $G$ in terms of the material derivative of the potential energy at equilibrium $W$ with respect to all possible regular virtual crack extensions. $W$ is formulable in terms of surface integrals; this fact in turn allows for a boundary-only formulation of its material derivative with respect to virtual crack extensions. The necessary step of computing the shape sensitivities of the boundary elastic variables is done by means of a derivative BIE. The latter results from a material differentiation of the primary elastic BIE, which is performed on a regularized (weakly singular) displacement BIE so that the process is mathematically sound. The unknowns of both primary and derivative BIEs are governed by the same integral operator, with obvious computational advantages. The present approach thus does not resort to any finite-difference evaluation of derivatives with respect to crack front perturbations.

The implementation of the present method, including the key technical step of constructing appropriate vector interpolation functions for the transformation velocity associated with a virtual crack extension, is discussed. Finally, in order to demonstrate the potential of the proposed approach, numerical results are presented on two mode I examples where reference results are available for comparison: the round bar with a circular internal crack and the semielliptical surface crack, in both cases under uniform tension.
\end{abstract}

KEYWORDS: regularized elastic BIE, material differentiation, variable domains, linear fracture mechanics, virtual crack extension. 


\section{Introduction}

One of the basic quantities involved in elastic fracture mechanics is the energy release rate $G(s)$, function of the arc length $s$ along the front $\partial \Gamma$ of a crack $\Gamma$ :

$$
\int_{\partial \Gamma} G(s) \delta \ell(s) \mathrm{d} s=-\delta W
$$

where $\delta W$ is the perturbation of the elastic potential energy at equilibrium $W$ induced by a crack front normal extension $\delta \ell$ and in the absence of load variation. For two-dimensional problems, $G$ is the value of the well-known path-independent $J$ integral. In linear fracture mechanics, $G$ is linked to the crack tip singularity of the stress field through the well-known Irwin formula [5]:

$$
G(s)=\frac{1+\nu}{2 \mu}\left[K_{I}^{2}(s)+K_{I I}^{2}(s)\right]+\frac{1}{2 \mu} K_{I I I}(s)
$$

where $K_{I}(s), K_{I I}(s), K_{I I I}(s)$ are the stress intensity factors (SIFs), $\mu$ and $\nu$ being respectively the shear modulus and Poisson ratio. Furthermore $G$ has a clear thermodynamical meaning (Nguyen [19]) and plays a central role for the prediction of crack extension according to Griffithtype criterions.

Thus the consideration of perturbations of $W$ under fictitious body changes associated to virtual crack extensions provides a computational tool for elastic crack analysis: this is the virtual crack extension approach. In the first numerical applications derivatives of $W$ are evaluated using small finite crack perturbations and finite differences (Hellen [12], Parks [21]). In later works (Delorenzi [9], Destuynder et al. [10], Mialon [15], Ohtsuka [20]), Suo \& Combescure [27] the concept of material differentiation is applied to $W$; this leads to rigorous formulations for $G$, starting from variational formulations of elasticity problems. This approach, sometimes known as the ' $\theta$-method' ( $\theta$ refers to the notation used in [10], [15] and herein for the transformation velocity), has led to FEM implementations (Wadier \& Malak [31]). The present paper aims at formulating a BIE version of the $\theta$-method.

A common factor shared by the present investigation subject and other situations where the geometrical domain is a primary variable, like shape optimization, geometrical inverse problems or free-boundary problems, is the presence of integral functionals being both directly (through the geometrical support of the integral) and indirectly (through mechanical fields which solve e.g. elastic boundary-value problems) shape-dependent, whose derivatives with respect to shape parameters, or more abstractly their domain derivatives, are of practical interest. It is known that finite-difference evaluations of gradients are both computationally expensive and prone to inaccuracies due to the known mathematically ill-posed nature of this operation (Tikhonov 
and Arsenin [30]). Hence it is natural to revert to analytical differentiation with respect to a variable domain. This concept has been discussed by several authors (see e.g. Haug et al. [11], Petryk and Mroz [22], in FEM-oriented contexts). Since in such problems the domain (and hence its boundary) is a primary variable, it is natural to consider the use of boundary integral formulations, which allow for the "minimal" modelling. The formulation of shape sensitivities in a BIE context may result from either the adjoint variable approach (Aithal \& Saigal [1], Bonnet [3], Choi \& Kwak [7], Meric [14]) or the direct differentiation approach (Barone \& Yang [2], Bonnet [4], Mukherjee \& Chandra [16], Zhang \& Mukherjee [32]).

In the present paper, the virtual crack extension approach is formulated for BIE analysis of crack problems, using the basic concepts developed inn [10], [15]. First some basic definitions and results about material differentiation are recalled. Then the definition of $G$, for a three-dimensional cracked solid, is given a boundary-only reformulation; as a result, $G$ solves a variational equation whose right-hand side depends linearly on the transformation velocity through the boundary shape sensitivities of elastic variables. In order to use only conventional displacement collocation BIE formulations, the framework of multiregion modelling is used. The formulation is general in terms of the fracture modes involved. Next, the governing BIE formulation for firstorder elastic shape sensitivities, whose solution is a necessary step for the numerical evaluation of $G$ in the present work, is given. Then, the numerical implementation of the method is described, and special attention is devoted to the key step of building appropriate (vector) interpolation functions for the transformation velocity in the vicinity of the crack front. Finally, numerical results are presented on two mode I examples where reference results are available for comparison: the round bar with a circular internal crack and the semi-elliptical surface crack, in both cases under uniform tension.

\section{First-order material derivative of a surface integral}

Let us consider, in the three-dimensional Euclidean space $\mathbb{R}^{3}$ equipped with a Cartesian orthonormal basis $\left(\boldsymbol{e}_{1}, \boldsymbol{e}_{2}, \boldsymbol{e}_{3}\right)$, a body $\Omega_{p}$ whose shape depends on a time-like parameter $p$ through a continuum kinematics-type lagrangian description, with the "initial" configuration $\Omega_{0}$ conventionally associated with $p=0$ :

$$
\boldsymbol{Y} \in \Omega_{0} \rightarrow \boldsymbol{y}=\boldsymbol{\Phi}(\boldsymbol{Y} ; p) \in \Omega_{p} \quad \text { where } \quad\left(\forall \boldsymbol{Y} \in \Omega_{0}\right) \boldsymbol{\Phi}(\boldsymbol{Y} ; 0)=\boldsymbol{Y}
$$

Throughout this paper lower-case boldface letters $\boldsymbol{x}, \boldsymbol{y}$ denote geometrical points on the current configuration $\Omega_{p}$. The diffeomorphism $\boldsymbol{\Phi}(\cdot ; p)$, or geometrical transformation, must possess a 
strictly positive Jacobian everywhere and for any $p \geq 0$. A given domain evolution considered as a whole admits many different representations (3), with different transformations $\boldsymbol{\Phi}$.

Material derivative of scalar or tensor fields. The transformation velocity $\boldsymbol{\theta}(\boldsymbol{y} ; p)$, defined by

$$
\boldsymbol{\theta}(\boldsymbol{y} ; p)=\boldsymbol{\Phi}_{, p}(\boldsymbol{Y} ; p) \quad \text { for } \quad \boldsymbol{y}=\boldsymbol{\Phi}(\boldsymbol{Y} ; p)
$$

is the (Eulerian representation for the) "velocity" of the "material" point wich coincides with the geometrical point $\boldsymbol{y}$ at "time" $p$.

Next, let $f(\boldsymbol{y} ; p)$ denote a scalar, vector or tensor field. The material derivative $\stackrel{\star}{f}(\boldsymbol{y} ; p)$ in the domain transformation $\boldsymbol{y}=\boldsymbol{\Phi}(\boldsymbol{Y} ; p)$ is defined (see e.g. Salençon [26]) as:

$$
\begin{aligned}
\stackrel{\star}{f}(\boldsymbol{y} ; p) & =\lim _{h \rightarrow 0} \frac{1}{h}[f(\boldsymbol{\Phi}(\boldsymbol{Y} ; p+h), p+h)-f(\boldsymbol{\Phi}(\boldsymbol{Y} ; p), p)] \\
& =f_{, p}(\boldsymbol{y} ; p)+\boldsymbol{\nabla} f(\boldsymbol{y} ; p) \cdot \boldsymbol{\theta}(\boldsymbol{y} ; p)
\end{aligned}
$$

where $\boldsymbol{\nabla}$ denotes the gradient with respect to "Eulerian" coordinates $\left(\boldsymbol{\nabla} f=\left(f_{, y_{i}}\right) \otimes \boldsymbol{e}_{i}\right)$. The material derivative of the gradient $\nabla f$ is thus given by:

$$
(\nabla f)^{\star}=\nabla \stackrel{\star}{f}-\nabla f . \nabla \theta
$$

It should be stressed that the thansformation $\boldsymbol{\Phi}$ is used to represent a continuous change of domain, each $\Omega_{p}$ being the geometrical support of a boundary value problem, as opposed to a material deformation of a given physical body; the "material" qualificative is thus only a convenient language abuse.

Material derivative of surface integrals. As shown in Appendix A, the material derivatives of the unit normal $\boldsymbol{n}$ and the surface differential element $\mathrm{d} S$ on a material surface $S_{p}=\boldsymbol{\Phi}(S ; p)$ are given by:

$$
\stackrel{\star}{\mathrm{d} S}=\operatorname{div} S \boldsymbol{\theta} \mathrm{d} S=D_{r} \theta_{r} \mathrm{~d} S \quad \stackrel{\star}{\boldsymbol{n}}=-\boldsymbol{n} \cdot \nabla_{S} \boldsymbol{\theta}=-n_{r} D_{j} \theta_{r} \boldsymbol{e}_{j}
$$

in terms of the surface gradient $\nabla_{S}$ and the surface divergence $\operatorname{div}_{S}$ :

$$
\begin{aligned}
& \nabla_{S} f=\nabla f-(\nabla f . \boldsymbol{n}) \boldsymbol{n}=\left(f_{, i}-n_{i} f_{, n}\right) \boldsymbol{e}_{i} \equiv\left(D_{i} f\right) \boldsymbol{e}_{i} \\
& \operatorname{div}_{S} \boldsymbol{u}=\operatorname{div} \boldsymbol{u}-(\boldsymbol{\nabla u} . \boldsymbol{n}) . \boldsymbol{n}=D_{i} u_{i}
\end{aligned}
$$

Then, for a generic surface integral $J(p)$ :

$$
J(p)=\int_{S_{p}} f(\boldsymbol{y}, p) \mathrm{d} S_{p}
$$


one has, using (7):

$$
\frac{d J}{d p}=\stackrel{\star}{J}=\int_{S_{p}}\left\{\stackrel{\star}{f} \mathrm{~d} S+f(\mathrm{~d} S)^{\star}\right\}=\int_{S_{p}}\left\{\stackrel{\star}{f}+f \operatorname{div}_{S} \boldsymbol{\theta}\right\} \mathrm{d} S
$$

Indeed $\stackrel{\star}{J}$ could be expressed in several other ways (see Petryk and Mroz [22]), but the above formula serves the purpose of the present paper.

\section{Formulation of the energy release rate in a BEM context}

Let the body $\Omega$ be elastic (shear modulus $\mu$, Poisson ratio $\nu$ ), its external boundary being split into $S_{T}$ (on which the traction vector $\boldsymbol{t}$ is given: $\boldsymbol{t}=\boldsymbol{t}^{D}$ ) and $S_{u}$ (on which the displacement $\boldsymbol{u}$ is given: $\left.\boldsymbol{u}=\boldsymbol{u}^{D}\right)$. No body forces are present. The potential energy of $\Omega$ at elastic equilibrium takes the value $W$ :

$$
W=\frac{1}{2} \int_{\Omega} \boldsymbol{\sigma}(\boldsymbol{u}): \boldsymbol{\varepsilon}(\boldsymbol{u}) \mathrm{d} V-\int_{S_{T}} \boldsymbol{t}^{D} \cdot \boldsymbol{u} \mathrm{d} S
$$

where $\boldsymbol{u}, \boldsymbol{\varepsilon}, \boldsymbol{\sigma}$ are the displacement, strain and stress fields solution to the elastic equilibrium problem with boundary data $\boldsymbol{u}^{D}, \boldsymbol{t}^{D}$.

Multiregion approach for a cracked elastic solid. Now suppose that a crack $\Gamma$, with upper and lower traction-free faces $\Gamma^{ \pm}$(figure 1) and unit normal $\boldsymbol{n}$ directed from $\Gamma^{-}$to $\Gamma^{+}$, is embedded in $\Omega$. In order to use only usual displacement BIE formulations, the so-called "multiregion approach" [8] is considered: $\Omega$ is split into two subdomains $\Omega^{+}, \Omega^{-}$separated by a surface $S$ containing the crack $\Gamma$ (figure 1). The cracked solid equilibrium is then formulated in terms of two elastic problems (one for each subdomain) with the following conditions on the external boundary:

$$
\left\{\begin{array}{llll}
\boldsymbol{u} & =\boldsymbol{u}^{D} & \text { on } S_{u}^{ \pm} & \\
\boldsymbol{t} & =\boldsymbol{t}^{D} & \text { on } S_{T}^{ \pm} & \text {for each subdomain } \Omega^{ \pm} \\
\boldsymbol{t} & = & 0 & \text { on } \Gamma^{ \pm}
\end{array}\right.
$$

and the following coupling conditions (perfect bonding between $\Omega^{+}$and $\Omega^{-}$outside the crack $\Gamma):$

$$
\left\{\begin{array}{l}
\boldsymbol{u}^{+}=\boldsymbol{u}^{-} \\
\boldsymbol{t}^{+}=-\boldsymbol{t}^{-}
\end{array} \text {on } S-\Gamma\right.
$$

Figure 1 should appear here. 
In the particular case of practical importance where $\Omega$ has a symmetry plane $\Pi$ containing a plane crack $\Gamma$ and the external loading $\boldsymbol{t}^{D}$ is symmetric with respect to $\Pi$, it is sufficient to consider the subproblem over $\Omega^{+}$(say), the coupling relations (13) being replaced by:

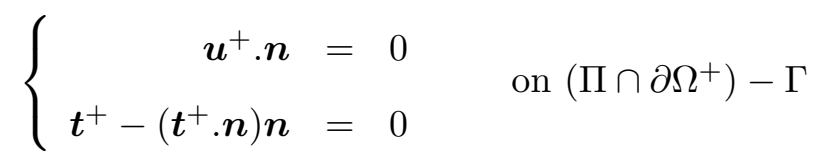

Definition of the energy release rate. The energy release rate $G$ associated with the cracked solid $\Omega$ and the loading $\boldsymbol{u}^{D}, \boldsymbol{t}^{D}$ is defined by (1), or, equivalently by:

$$
\int_{\partial \Gamma} G \theta_{\nu} \mathrm{d} s=-\stackrel{\star}{W} \quad \forall \boldsymbol{\theta} \in \Theta
$$

where $\boldsymbol{\nu}$ is the unit normal to the crack front $\partial \Gamma$ exterior to $\Gamma$ and tangent to $\Gamma$. Also, $\Theta$ denotes the set of virtual crack extensions, that is, those transformation velocities $\boldsymbol{\theta}$ associated with geometrical transformations $\mathbf{\Phi}(\cdot ; p)$ which describe a crack extension: one has

$$
\boldsymbol{\theta} . \boldsymbol{n}=0 \quad \text { on } \Gamma \quad \boldsymbol{\theta}=\mathbf{0} \text { on } S_{u}, S_{T}
$$

Thus only regular virtual crack extensions (i.e. without kinking) are considered. Moreover, in eq. (15) the variation $\stackrel{\star}{W}$ of $W$ is taken for constant loading $\left(\boldsymbol{u}^{D}, \boldsymbol{t}^{D}\right)$ so that one has, within the multiregion framework:

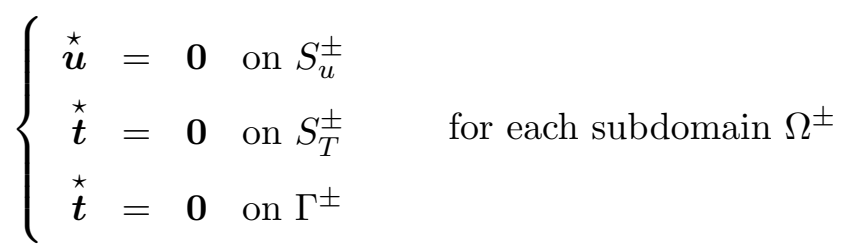

with the coupling conditions:

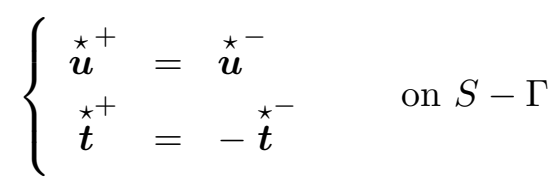

Boundary-only formulation of the energy release rate. Now the value $W$ of the potential energy at equilibrium, eq. (11), can be reformulated using boundary integrals: applying the divergence-flux theorem to the domain integral in (11) and taking into account the equilibrium equation $\operatorname{div} \boldsymbol{\sigma}=0$ gives the well-known alternative expression:

$$
W=\frac{1}{2} \int_{S_{u}} \boldsymbol{t} \cdot \boldsymbol{u}^{D} d S-\frac{1}{2} \int_{S_{T}} \boldsymbol{t}^{D} \cdot \boldsymbol{u} \mathrm{d} S
$$

The variation $\stackrel{\star}{W}$ of $W$ in a crack extension thus stems from application of formula (10) to the above equation. Since $\boldsymbol{\theta} \in \Theta$, eq. (11), and accounting for the boundary conditions $(17), \stackrel{\star}{W}$ is 
finally expressed in terms of the material derivative of the boundary elastic variables as follows:

$$
\stackrel{\star}{W}=\frac{1}{2} \int_{S_{u}} \stackrel{\star}{\boldsymbol{t}} \cdot \boldsymbol{u}^{D} \mathrm{~d} S-\frac{1}{2} \int_{S_{T}} \boldsymbol{t}^{D} \cdot \stackrel{\star}{\boldsymbol{u}} \mathrm{d} S
$$

The derivatives $(\stackrel{\star}{\boldsymbol{u}}, \stackrel{\star}{\boldsymbol{t}})$ depend linearly on $\boldsymbol{\theta}$, through a derivative BIE to be discussed in the next section, which has to be solved for all $\boldsymbol{\theta}$ in (a finite-dimensional subspace of) $\Theta$.

Thus, incorporation of the variant expression (19) for $\stackrel{\star}{W}$ into the variational equation (15) leads to a boundary-only approach to the computation of $G(s)$. Note that it is known (see e.g. Mialon [15]) that the right-hand side $-\stackrel{\star}{W}$ of $(15)$ depends ultimately only on the normal crack front virtual extension $\left.\theta_{\nu}\right|_{\partial \Gamma}$, not on any particular continuation of $\boldsymbol{\theta}$ outside $\partial \Gamma$, although this fact is not apparent. This is an important consideration because the present use of Lagrangiantype material differentiation necessarily requires such continuation of $\boldsymbol{\theta}$.

\section{First-order elastic shape sensitivity formulation}

The intermediary step of determining the material derivatives $(\stackrel{\star}{\boldsymbol{u}}, \stackrel{\star}{\boldsymbol{t}})$ for every $\boldsymbol{\theta} \in \Theta$ is now discussed for the sake of completeness. Governing BIEs for the first- and second-order elastic sensitivities are investigated in more detail in [4].

Governing regularized elastic BIE. Any elastostatic state on $\Omega$ with zero body forces can be characterized by the boundary variables governed by the following regularized displacement BIE (Rizzo and Shippy [25], Bui et al. [6]):

$$
\int_{\partial \Omega}\left[u_{i}(\boldsymbol{y})-u_{i}(\boldsymbol{x})\right] n_{j}(\boldsymbol{y}) \Sigma_{i j}^{k}(\boldsymbol{x}, \boldsymbol{y}) \mathrm{d} S_{y}-\int_{\partial \Omega} t_{i}(\boldsymbol{y}) U_{i}^{k}(\boldsymbol{x}, \boldsymbol{y}) \mathrm{d} S_{y}=0
$$

or, introducing for later convenience an abbreviated notation:

$$
I_{1}(\boldsymbol{x}, \boldsymbol{u})-I_{2}(\boldsymbol{x}, \boldsymbol{t})=0
$$

using the Kelvin infinite-space fundamental displacement and elastic stress tensor:

$$
\begin{aligned}
U_{i}^{k}(\boldsymbol{x}, \boldsymbol{y}) & =-\frac{1}{16 \pi \mu(1-\nu) r}\left[(3-4 \nu) \delta_{i k}+r_{, i} r_{, k}\right] \\
\Sigma_{i j}^{k}(\boldsymbol{x}, \boldsymbol{y}) & =-\frac{1}{8 \pi(1-\nu) r^{2}}\left[(1-2 \nu)\left(\delta_{i k} r_{, j}+\delta_{j k} r_{, i}-\delta_{i j} r_{, k}\right)+3 r_{, i} r_{, j} r_{, k}\right]
\end{aligned}
$$

created at $\boldsymbol{y} \in \mathbb{R}^{3}$ by a unit point force applied at the collocation point $\boldsymbol{x}$ along the $\boldsymbol{e}_{k}$-direction. Also, $r=|y-x|$ is the Euclidian distance between $\boldsymbol{y}, \boldsymbol{x}$ and ()$_{, i}$ denotes a partial derivative with respect to $y_{i}$. 
The BIE (20) holds for any collocation point $\boldsymbol{x} \in \mathbb{R}^{3}$. The displacement $\boldsymbol{u}$ is required to be Hölder-continuous at $\boldsymbol{x}$ when $\boldsymbol{x}$ is taken on $\partial \Omega[13]$ :

$$
\exists C>0, \exists \alpha \in] 0,1] \text { such that }|u(y)-u(x)| \leq C|y-x|^{\alpha}
$$

for the regularization provided by the presence of the term $[\boldsymbol{u}(\boldsymbol{y})-\boldsymbol{u}(\boldsymbol{x})]$ in (20) to be effective.

First-order sensitivity formulation. A small perturbation of the domain $\Omega=\Omega_{p}$ associated to a small increment $d p$ induces a perturbation of the elastostatic state $(\boldsymbol{u}, \boldsymbol{t})$, which may be expressed in terms of the first-order material derivatives $(\stackrel{\star}{\boldsymbol{u}}, \stackrel{\star}{\boldsymbol{t}})$ :

$$
\delta \boldsymbol{u}=\stackrel{\star}{\boldsymbol{u}} d p+o(d p) \quad \delta \boldsymbol{t}=\stackrel{\star}{\boldsymbol{t}} d p+o(d p)
$$

This idea is consistent with the present BIE framework: the boundary $\partial \Omega_{p}$ of a material domain $\Omega_{p}$ is itself material, hence $\left.(\stackrel{\star}{\boldsymbol{u}}, \stackrel{\star}{\boldsymbol{t}})\right|_{\partial \Omega_{p}}$ are completely determined by the knowledge of $\left.(\boldsymbol{u}, \boldsymbol{t})\right|_{\partial \Omega_{p+d p}}$ for the neighbouring perturbed boundary configurations. In other words, the material derivatives $\left.(\stackrel{\star}{\boldsymbol{u}}, \boldsymbol{t})\right|_{\partial \Omega_{p}}$ are taken while "staying on the moving boundary". The BIE (21) governs any elastostatic state defined on $\Omega_{p}$ for any $p \geq 0$ such that $\boldsymbol{\Phi}(\cdot ; p)$ is defined. Thus taking the material derivative using formula (10) yields the governing BIE satisfied by any possible pair $(\stackrel{\star}{\boldsymbol{u}}, \boldsymbol{t})$. This operation is now carried out, with the assumption that the collocation point $\boldsymbol{x}$ also follows the material transformation (3).

First, a direct application of formula (10) to the integral operators $I_{1}, I_{2}$ gives :

$$
\begin{aligned}
& {\stackrel{\star}{I_{1}}}(\boldsymbol{x}, \boldsymbol{u})=I_{1}(\boldsymbol{x}, \stackrel{\star}{\boldsymbol{u}})+J_{1}(\boldsymbol{x}, \boldsymbol{u} ; \boldsymbol{\theta}) \\
& {\stackrel{\star}{I_{2}}}_{2}(\boldsymbol{x}, \boldsymbol{t})=I_{2}(\boldsymbol{x}, \stackrel{\star}{\boldsymbol{t}})+J_{2}(\boldsymbol{x}, \boldsymbol{t} ; \boldsymbol{\theta})
\end{aligned}
$$

with

$$
\begin{aligned}
J_{1}(\boldsymbol{x}, \boldsymbol{u} ; \boldsymbol{\theta}) & \left.=\int_{\partial \Omega}\left[u_{i}(\boldsymbol{y})-u_{i}(\boldsymbol{x})\right]\left\{n_{j}(\boldsymbol{y}) \stackrel{\star}{\Sigma_{i j}^{k}}(\boldsymbol{x}, \boldsymbol{y})+\Sigma_{i j}^{k}(\boldsymbol{x}, \boldsymbol{y}) \stackrel{n}{n}_{j}(\boldsymbol{y})+n_{j}(\boldsymbol{y}) D_{r} \theta_{r}(\boldsymbol{y})\right]\right\} \mathrm{d} S_{y} \\
& =\int_{\partial \Omega}\left[u_{i}(\boldsymbol{y})-u_{i}(\boldsymbol{x})\right]\left\{n_{j}(\boldsymbol{y})\left[\left[\theta_{r}(\boldsymbol{y})-\theta_{r}(\boldsymbol{x})\right]\right] \Sigma_{i j, r}^{k}(\boldsymbol{x}, \boldsymbol{y})\right. \\
& \left.-\Sigma_{i j}^{k}(\boldsymbol{x}, \boldsymbol{y})\left[n_{r}(\boldsymbol{y}) D_{j} \theta_{r}(\boldsymbol{y})-n_{j}(\boldsymbol{y}) D_{r} \theta_{r}(\boldsymbol{y})\right]\right\} \mathrm{d} S_{y} \\
& =\int_{\partial \Omega}\left[u_{i}(\boldsymbol{y})-u_{i}(\boldsymbol{x})\right]\left\{\left[\left[\theta_{r}(\boldsymbol{y})-\theta_{r}(\boldsymbol{x})\right]\right] n_{j}(\boldsymbol{y}) \Sigma_{i j, r}^{k}(\boldsymbol{x}, \boldsymbol{y})-\Sigma_{i j}^{k}(\boldsymbol{x}, \boldsymbol{y}) D_{r j} \theta_{r}(\boldsymbol{y})\right\} \mathrm{d} S_{y} \\
J_{2}(\boldsymbol{x}, \boldsymbol{t} ; \boldsymbol{\theta})= & \int_{\partial \Omega} t_{i}(\boldsymbol{y})\left\{\stackrel{\star}{U}_{i}^{k}(\boldsymbol{x}, \boldsymbol{y})+U_{i}^{k}(\boldsymbol{x}, \boldsymbol{y}) D_{r} \theta_{r}(\boldsymbol{y})\right\} \mathrm{d} S_{y} \\
= & \int_{\partial \Omega} t_{i}(\boldsymbol{y})\left\{\left[\left[\theta_{r}(\boldsymbol{y})-\theta_{r}(\boldsymbol{x})\right]\right] U_{i, r}^{k}(\boldsymbol{x}, \boldsymbol{y})+U_{i}^{k}(\boldsymbol{x}, \boldsymbol{y}) D_{r} \theta_{r}(\boldsymbol{y})\right\} \mathrm{d} S_{y}
\end{aligned}
$$


where formulas (7) have been taken into account, together with the relations

$$
\begin{aligned}
{\stackrel{\star \star}{\Sigma_{i j}^{k}}}_{i j}(\boldsymbol{x}, \boldsymbol{y}) & =\left[\left[\theta_{r}(\boldsymbol{y})-\theta_{r}(\boldsymbol{x})\right]\right] \Sigma_{i j, r}^{k}(\boldsymbol{x}, \boldsymbol{y}) \\
\stackrel{\star}{U}_{i}^{k}(\boldsymbol{x}, \boldsymbol{y}) & =\left[\left[\theta_{r}(\boldsymbol{y})-\theta_{r}(\boldsymbol{x})\right]\right] U_{i, r}^{k}(\boldsymbol{x}, \boldsymbol{y})
\end{aligned}
$$

which stem from (6) and the fact that the Kelvin solution does not depend on $p$. The tangential differential operator $D_{r j}$ is defined as:

$$
D_{r j} f=n_{r} D_{j} f-n_{j} D_{r} f=n_{r} f_{, j}-n_{j} f_{, r}=e_{r s j} e_{a b j} n_{a} f_{, b}
$$

The integrals in (27) are convergent under the requirements (24) and

$$
\exists C>0 \text { such that }|\boldsymbol{\theta}(\boldsymbol{y})-\boldsymbol{\theta}(\boldsymbol{x})| \leq C|y-x|
$$

Note that the latter is weaker than the $C^{1}$-continuity at $\boldsymbol{x}$; for instance the appearance of an edge or corner out of an initially smooth $\partial \Omega$ is allowed.

Finally, it is convenient to rearrange expression $(27)$ for $J_{1}(\boldsymbol{x}, \boldsymbol{u} ; \boldsymbol{\theta})$ further, using the following variant of Stokes' formula:

$$
\int_{S} D_{r j} f \mathrm{~d} S=e_{r s j} \int_{S} e_{a b j} n_{a} f_{, b} \mathrm{~d} S=0
$$

which is valid for any piecewise regular closed surface $S$ and continuous, piecewise differentiable f. Then:

$$
\begin{aligned}
\int_{\partial \Omega}\left[u_{i}(\boldsymbol{y})-u_{i}(\boldsymbol{x})\right]\left[\left[\theta_{r}(\boldsymbol{y})-\theta_{r}(\boldsymbol{x})\right]\right] n_{j}(\boldsymbol{y}) \sum_{i j, r}^{k}(\boldsymbol{x}, \boldsymbol{y}) \mathrm{d} S_{y} \\
=-\int_{\partial \Omega}\left[u_{i}(\boldsymbol{y})-u_{i}(\boldsymbol{x})\right]\left[\left[\theta_{r}(\boldsymbol{y})-\theta_{r}(\boldsymbol{x})\right]\right]\left\{D_{r j} \Sigma_{i j}^{k}(\boldsymbol{x}, \boldsymbol{y})+n_{r}(\boldsymbol{y}) \Sigma_{i j, j}^{k}(\boldsymbol{x}, \boldsymbol{y})\right\} \mathrm{d} S_{y} \\
=-\int_{\partial \Omega}\left[u_{i}(\boldsymbol{y})-u_{i}(\boldsymbol{x})\right]\left[\left[\theta_{r}(\boldsymbol{y})-\theta_{r}(\boldsymbol{x})\right]\right] D_{r j} \sum_{i j}^{k}(\boldsymbol{x}, \boldsymbol{y}) \mathrm{d} S_{y} \\
=\int_{\partial \Omega} D_{r j}\left\{\left[u_{i}(\boldsymbol{y})-u_{i}(\boldsymbol{x})\right]\left[\left[\theta_{r}(\boldsymbol{y})-\theta_{r}(\boldsymbol{x})\right]\right]\right\} \Sigma_{i j}^{k}(\boldsymbol{x}, \boldsymbol{y}) \mathrm{d} S_{y}
\end{aligned}
$$

where the differential operator $D_{r j}$ is understood as acting on the variable $\boldsymbol{y}$ and use has been made of the equilibrium equation $\Sigma_{i j, j}^{k}=0(\boldsymbol{y} \neq \boldsymbol{x})$. Substitution of eq. (30) into (27) yields:

$$
J_{1}(\boldsymbol{x}, \boldsymbol{u} ; \boldsymbol{\theta})=\int_{\partial \Omega}\left[\left[\theta_{r}(\boldsymbol{y})-\theta_{r}(\boldsymbol{x})\right]\right] D_{r j} u_{i}(\boldsymbol{y}) \Sigma_{i j}^{k}(\boldsymbol{x}, \boldsymbol{y}) \mathrm{d} S_{y}
$$

which is more compact than (27).

Derivative BIE. The material derivatives $(\stackrel{\star}{\boldsymbol{u}}, \stackrel{\star}{\boldsymbol{t}})$ associated with any elastostatic state $(\boldsymbol{u}, \boldsymbol{t})$ on $\Omega=\Omega_{p}$ are governed by the following derivative BIE:

$$
I_{1}(\boldsymbol{x}, \stackrel{\star}{\boldsymbol{u}})-I_{2}(\boldsymbol{x}, \stackrel{\star}{\boldsymbol{t}})=-J_{1}(\boldsymbol{x}, \boldsymbol{u} ; \boldsymbol{\theta})+J_{2}(\boldsymbol{x}, \boldsymbol{t} ; \boldsymbol{\theta})
$$


where $J_{1}, J_{2}$ are given by eqns. (31), (28). This result holds for any collocation point $\boldsymbol{x} \in \mathbb{R}^{3}$, hence it defines both an integral equation (with $\boldsymbol{x} \in \partial \Omega_{p}$ ) or an integral representation of $\stackrel{\star}{\boldsymbol{u}}(\boldsymbol{x} ; p)$ (with $\boldsymbol{x}$ interior to $\Omega_{p}$ ). Its right-hand side is explicitly, and linearly, dependent on the transformation velocity $\boldsymbol{\theta}$. The derivative BIE (32) is weakly singular under the requirements (24), (29), and this is also true for all intermediate calculations used for its derivation.

Comments about the derivative BIE. Firstly, it is apparent from (21) and (32) that the same integral operator govern the primary variables $(\boldsymbol{u}, \boldsymbol{t})$ and their material derivatives. Also, for a given transformation velocity $\boldsymbol{\theta}$, the derivative BIE (32) has many solutions $(\stackrel{\star}{\boldsymbol{u}}, \stackrel{\star}{\boldsymbol{t}}$ ). In order to ensure uniqueness of $(\stackrel{\star}{\boldsymbol{u}}, \stackrel{\boldsymbol{t}}{)})$, one has in addition to specify how the boundary conditions associated with the elastic problem evolve with $\Omega_{p}$. It is simplest to assume that the transformation $\mathbf{\Phi}(\cdot ; p)$ which describes a given change of domain is chosen so that the Dirichlet and Neumann parts $S_{u, p}, S_{T, p}$ of $\partial \Omega_{p}$ are respectively transformed into the Dirichlet and Neumann parts $S_{u, p+d p}, S_{T, p+d p}$ of $\partial \Omega_{p+d p}$, so that $(\boldsymbol{u}, \stackrel{\star}{\boldsymbol{u}})$ and $(\boldsymbol{t}, \boldsymbol{\star})$ are unknown (resp. known) over the same portions of the boundary. Then $(\stackrel{\star}{\boldsymbol{u}}, \hat{t})$ are linear forms over $\boldsymbol{\theta}$ provided their prescribed parts are themselves linear forms over $\boldsymbol{\theta}$, thus the unknown parts of $(\boldsymbol{u}, \boldsymbol{t})$ and of $(\stackrel{\star}{\boldsymbol{u}}, \stackrel{\star}{\boldsymbol{t}})$ share the same integral governing operator. This remark constitutes an important computational advantage: the discretized integral operator is built and factored only once, in the course of a boundary element solution to (21), then later repeatedly reused for the numerical solution of the derivative BIE (32).

\section{BEM formulation}

The surfaces $\partial \Omega^{+}$and $\partial \Omega^{-}$are approximated by classical boundary elements. Each element $E_{e}$ is mapped on a parent element $\Delta_{e}$, which is either the square $\left(\xi_{1}, \xi_{2}\right) \in[-1,1]^{2}$ or the triangle $0 \leq \xi_{1}+\xi_{2} \leq 1$, in terms of NU shape functions $N_{k}$ and nodes $\boldsymbol{y}^{k}$ (in global numbering); isoparametric interpolation of the elastic variables $(\boldsymbol{u}, \boldsymbol{t})$ is considered, so that:

$$
\left\{\begin{array}{l}
\boldsymbol{y} \\
\boldsymbol{u}(\boldsymbol{y}) \\
\boldsymbol{t}(\boldsymbol{y})
\end{array}\right\}=\sum_{k=1}^{N U} N_{k}(\boldsymbol{\xi})\left\{\begin{array}{c}
\boldsymbol{y} \\
\boldsymbol{u}^{k} \\
\boldsymbol{t}^{k}
\end{array}\right\} \quad \boldsymbol{\xi}=\left(\xi_{1}, \xi_{2}\right) \in \Delta_{e}
$$

We denote respectively by $\boldsymbol{a}_{\alpha}(\boldsymbol{\xi})(\alpha=1,2)$ and $\boldsymbol{n}(\boldsymbol{\xi})$ the natural basis of the tangent plane and the unit normal at $\boldsymbol{y}(\boldsymbol{\xi})$ :

$$
\boldsymbol{a}_{\alpha}(\boldsymbol{\xi})=\sum_{k=1}^{N U} N_{k, \alpha}(\boldsymbol{\xi}) \boldsymbol{y}^{k} \quad(\alpha=1,2) \quad \boldsymbol{n}(\boldsymbol{y})=\frac{1}{\sqrt{J}}(\boldsymbol{a} \wedge \boldsymbol{b})
$$


with

$$
J=g_{11} g_{22}-g_{12}^{2}=\left\|\boldsymbol{a}_{1} \wedge \boldsymbol{a}_{2}\right\|^{2} \quad g_{\alpha \beta}=\boldsymbol{a}_{\alpha} \cdot \boldsymbol{a}_{\beta} \quad(\alpha, \beta=1,2)
$$

The three basic steps involved in the computation of $G$ using the present approach are now described. Nine-noded quadrilateral elements were used for the numerical exemples presented in this paper.

1 - Solution of the primary BIE. The BIE (20) is discretized along the lines outlined above. The coupled elastostatic problems on $\Omega=\Omega^{+} \cup \Omega^{-}$are numerically solved with boundary conditions (12) and continuity conditions (13). If $\Omega$ is symmetric with respect to a plane $\Pi$ and if $\Gamma \subset \Pi$, only one boundary value problem is to be solved, the continuity conditions becoming (14). This step involves the building of the usual BEM discrete linear equation:

$$
[\boldsymbol{A}]\{\boldsymbol{u}\}+[\boldsymbol{B}]\{\boldsymbol{t}\}=\{\boldsymbol{0}\}
$$

After appropriate column switches, one obtains the governing linear systems of equations on the vector $\{\boldsymbol{v}\}$ of elastostatic unknowns:

$$
[\boldsymbol{K}]\{\boldsymbol{v}\}=\left\{\boldsymbol{g}^{0}\right\}
$$

2 - Solution of the derivative BIE. This step involves the construction of a discrete set of admissible transformation velocity fields $\boldsymbol{\theta} \in \Theta$. Denote by $E(\partial \Gamma)$ the set of boundary elements adjacent to the crack front $\partial \Gamma$ and let $\boldsymbol{A}^{1}, \ldots, \boldsymbol{A}^{\mathrm{NC}}$ be the NC mesh nodes located on $\partial \Gamma$ (figure 2). The local numbering of nodes on each element $E \in E(\partial \Gamma)$ is arranged so that the curve $\left(\xi_{2}=-1\right)$, associated with the nodes $1,2,3$, is located on $\partial \Gamma$. In order to take into account the known fact that $\stackrel{\star}{W}$ ultimately depends only on the normal extension velocity $\theta_{\nu}$ of the crack front, transformation velocities of the following form are introduced:

$$
\boldsymbol{\theta}(\boldsymbol{y})=\sum_{k=1}^{\mathrm{NC}} \theta_{k} \boldsymbol{B}^{k}(\boldsymbol{\xi}) \quad \boldsymbol{y}=\boldsymbol{y}(\boldsymbol{\xi})
$$

in terms of NC scalar nodal values $\theta_{k}=\theta_{\nu}\left(\boldsymbol{A}^{k}\right)$ of $\theta_{\nu}$ and vector interpolation functions $\boldsymbol{B}^{k}$. Many choices are possible for the $\boldsymbol{B}^{k}$; here they are defined so that $\boldsymbol{\theta}(\boldsymbol{y})=0$ outside $E(\partial \Gamma)$ and, on any element $E_{e} \in E(\partial \Gamma)$ :

$$
\begin{array}{ll}
\boldsymbol{B}^{k}\left(\xi_{1}, 1\right)=0 & \xi_{1} \in[-1,1] \\
\boldsymbol{B}^{k}\left(\eta_{1}^{\ell},-1\right)=\delta_{k \ell} \boldsymbol{\nu}\left(\eta_{1}^{\ell},-1\right) & \ell=1,2,3
\end{array}
$$


where $\boldsymbol{\eta}^{\ell} \in \Delta_{e}$ is the antecedent of the crack front node $\boldsymbol{A}^{\ell} \in E_{e} \subset E(\partial \Gamma)$ and the local numbering $k=1,2,3$ is used. Defining a continuation $\boldsymbol{\nu}(\boldsymbol{\xi})$ of the unit normal to $\partial \Gamma$ as follows:

$$
\boldsymbol{\nu}=\frac{1}{\left|a_{1}\right|}\left(\boldsymbol{a}_{\mathbf{1}} \wedge \boldsymbol{n}\right)=\frac{g_{12}}{\sqrt{g_{11} J}} \boldsymbol{a}_{1}-\frac{g_{11}}{\sqrt{g_{11} J}} \boldsymbol{a}_{2}
$$

the $\boldsymbol{B}^{k}(\boldsymbol{\xi})$ (in local numbering) are defined as:

$$
B^{k}(\boldsymbol{\xi})=f\left(\xi_{2}\right) S_{k}\left(\xi_{1}\right) \boldsymbol{\nu}(\boldsymbol{\xi})
$$

where $S_{1}, S_{2}, S_{3}$ are the classical one-dimensional quadratic shape functions

$$
S_{1}\left(\xi_{1}\right)=\xi_{1}\left(\xi_{1}-1\right) / 2 \quad S_{2}\left(\xi_{1}\right)=1-\xi_{1}^{2} \quad S_{3}\left(\xi_{1}\right)
$$

and $f$ is a continuous function such that $f(1)=0, f(-1)=1$. It is suggested to use $f(\xi)=$ $\left(3-2 \xi-\xi^{2}\right) / 4$ with quarter-node elements (this allows for a linear variation of the factor $f$ in the physical space) or $f(\xi)=(1-\xi) / 2$ with ordinary elements. The definition (40) satisfy the constraints (38); moreover the transformation velocities $\boldsymbol{\theta}$, eq. (37), are continuous over $\partial \Omega^{ \pm}$ and satisfy the requirements (16). The interpolation of $\theta_{\nu}$ on $\Gamma$ takes the form of a standard one-dimensional interpolation:

$$
\theta_{\nu}(\boldsymbol{y})=\sum_{k=1}^{N C} \theta_{k} S_{k}\left(\xi_{1}\right)
$$

Figure 2 should appear here.

Figure 3 should appear here.

Figure 4 should appear here.

The definition (37)-(40) is then substituted into the derivative BIE (32). Due to the linearity of the right hand side of (32) with respect to $\boldsymbol{\theta}$, it is sufficient to compute the solutions $\left(\stackrel{\star}{\boldsymbol{U}}, \stackrel{\boldsymbol{T}}{k}^{k}\right)$ to (32) for the particular choices $\boldsymbol{\theta}=\boldsymbol{B}^{k}$, so that the pair $(\stackrel{\star}{\boldsymbol{u}}, \stackrel{\star}{\boldsymbol{t}})$ associated with $\boldsymbol{\theta}$ defined by (37) is given by

$$
\stackrel{\star}{\boldsymbol{u}}=\sum_{k=1}^{\mathrm{NC}} \theta_{k} \stackrel{\star}{\boldsymbol{U}}^{k} \quad \stackrel{\star}{\boldsymbol{t}}=\sum_{k=1}^{\mathrm{NC}} \theta_{k} \stackrel{\star \star}{\boldsymbol{T}}^{k}
$$

The pair $\left(\stackrel{\star}{\boldsymbol{U}}^{k}, \stackrel{\star \star T}{\boldsymbol{T}}^{k}\right)$ satisfies the matrix relation:

$$
[\boldsymbol{A}]\left\{\stackrel{\star}{\boldsymbol{U}}^{k}\right\}+[\boldsymbol{B}]\left\{\stackrel{\star}{*}^{k}\right\}=\left\{\boldsymbol{f}^{1}\left(\boldsymbol{u}, \boldsymbol{t} ; \boldsymbol{B}^{k}\right)\right\}
$$

where the right-hand side $\left\{\boldsymbol{f}^{1}\right\}\left(\boldsymbol{u}, \boldsymbol{t} ; \boldsymbol{B}^{k}\right)$ comes from the discretization of the right-hand side $J_{2}(\boldsymbol{x}, \boldsymbol{t} ; \boldsymbol{\theta})-J_{1}(\boldsymbol{x}, \boldsymbol{u} ; \boldsymbol{\theta})$ of eq. (32) with $\boldsymbol{\theta}=\boldsymbol{B}^{k}$. The above equation, together with the 
homogeneous boundary conditions (17), leads to the governing linear systems of equations for the vector $\{\stackrel{\star}{\boldsymbol{v}}\}$ of unknown derivatives:

$$
[\boldsymbol{K}]\{\stackrel{\star}{\boldsymbol{v}}\}=\left\{\boldsymbol{f}^{1}\left(\boldsymbol{u}, \boldsymbol{t} ; \boldsymbol{B}^{k}\right)\right\}
$$

The same matrix $[\boldsymbol{K}]$ appears in (36) and (44), because the present construction of $\boldsymbol{\theta}$ is such that the Dirichlet and Neumann parts $S_{u}^{ \pm}, S_{T}^{ \pm}$remain fixed and thus are material surfaces.

3 - Solution of the governing variational equation for $G$. The energy release rate is interpolated, similarly to $\left.\theta_{\nu}\right|_{\partial \Gamma}$, as:

$$
G(s)=\sum_{k=1}^{N C} G_{k} S_{k}\left(\xi_{2}\right)
$$

$G_{k}$ being the nodal values $G\left(\boldsymbol{A}^{k}\right)$. Then $(\stackrel{\star}{\boldsymbol{u}}, \boldsymbol{t})$, eq. (43), are substituted into the expression (19) of $\stackrel{\star}{W}$, so that the discretized form of the variational equation (15) reads:

$$
\begin{aligned}
& \text { find } G_{k} \quad \forall \theta_{m}(1 \leq m \leq \mathrm{NC}) \\
& \qquad \theta_{m}\left\{G_{k} \int_{\Gamma} S_{k}(s) S_{m}(s) \mathrm{d} s+\frac{1}{2} \int_{S_{u}} \stackrel{\star}{\boldsymbol{T}}_{m} \cdot \boldsymbol{u}^{D} \mathrm{~d} S-\frac{1}{2} \int_{S_{T}} \boldsymbol{t}^{D} \cdot \stackrel{\star}{\boldsymbol{U}}_{m} \mathrm{~d} S\right\}=0
\end{aligned}
$$

which, equating to zero the coefficient of each $\theta_{m}$, leads to a linear (symmetric and banded) matrix equation for the NC unknowns $G_{k}$.

Evaluation of tangential differential operators. The derivative BIE (32) makes use of tangential differential operators, for which $\mathrm{BE}$ implementation-oriented expressions are now given. First, from classical differential geometry, the surface gradient $\nabla_{S} f$ of a scalar function expressed in terms of the variable $\boldsymbol{\xi} \in \Delta_{e}$ is given by:

$$
\nabla_{S} f=\left(D_{r} f\right) \boldsymbol{e}_{r} \quad \text { with } \quad D_{r} f=f_{, \alpha} g^{\alpha \beta}\left(\boldsymbol{a}_{\boldsymbol{\beta}} \cdot \boldsymbol{e}_{\boldsymbol{r}}\right)
$$

where $g^{\alpha \beta}$ are contravariant components of $\boldsymbol{g}: g^{\alpha \gamma} g_{\gamma \beta}=\delta_{\beta}^{\alpha}$, see eq. (35). Equation (47) also holds for cartesian components of vector or tensor fields, so that:

$$
\begin{aligned}
D_{r} \theta_{r} & =\theta_{r, \alpha} g^{\alpha \beta}\left(\boldsymbol{a}_{\boldsymbol{\beta}} \cdot \boldsymbol{e}_{\boldsymbol{r}}\right) \\
D_{r j} u_{i} \mathrm{~d} S_{y} & =\epsilon_{r j q}\left[u_{i, 1}\left(\boldsymbol{a}_{\mathbf{2}} \cdot \boldsymbol{e}_{\boldsymbol{q}}\right)-u_{i, 2}\left(\boldsymbol{a}_{\mathbf{1}} \cdot \boldsymbol{e}_{\boldsymbol{q}}\right)\right] \mathrm{d} \xi
\end{aligned}
$$

Numerical evaluation of singular integrals. Singular integrals over an element $E_{e}$ occur if $\boldsymbol{x} \in E_{e}$. Following a common practice in $\operatorname{BEM}[24]$, set $\xi_{1}=\rho \cos \varphi, \xi_{2}=\rho \sin \varphi$, where $\boldsymbol{\eta}=\left(\eta_{1}, \eta_{2}\right)$ denote the antecedent of $\boldsymbol{x}$ on $\Delta_{e}$. Then:

$$
\mathrm{d} \xi=\rho d \rho d \varphi
$$


Moreover, for any shape function $N(\boldsymbol{\xi})$, one can introduce modified shape functions $\hat{N}(\rho, \varphi ; \boldsymbol{\eta})$, regular at $\rho=0$ and such that:

$$
N(\boldsymbol{\xi})-N(\boldsymbol{\eta})=\rho \hat{N}^{q}(\rho, \varphi ; \boldsymbol{\eta})
$$

so that one has from (33):

$$
r=|x-y|=\rho\left|\sum_{k=1}^{N} \hat{N}^{k}(\rho, \varphi ; \boldsymbol{\eta}) \boldsymbol{y}^{k}\right| \equiv \rho \hat{r}(\rho, \varphi ; \boldsymbol{\eta})
$$

and $\hat{r}(\rho, \varphi ; \boldsymbol{\eta}) \neq 0$. Consequently, since $U^{k}(\boldsymbol{x}, \boldsymbol{y}), \Sigma^{k}(\boldsymbol{x}, \boldsymbol{y})$ behave like $r^{-1}, r^{-2}$ respectively:

$$
U_{i}^{k}(\boldsymbol{x}, \boldsymbol{y})=\frac{1}{\rho} \hat{U}_{i}^{k}(\rho, \varphi ; \boldsymbol{\eta}) \quad \Sigma_{i j}^{k}(\boldsymbol{x}, \boldsymbol{y})=\frac{1}{\rho^{2}} \hat{\Sigma}_{i j}^{k}(\rho, \varphi ; \boldsymbol{\eta})
$$

where $\hat{U}_{i}^{k}(\rho, \varphi ; \boldsymbol{\eta})$ and $\hat{\Sigma}_{i j}^{k}(\rho, \varphi ; \boldsymbol{\eta})$ are regular at $\rho=0$. Finally, using the modified interpolation functions $\hat{N}_{k}, \hat{\boldsymbol{B}}^{k}$ associated with the interpolations (33) of $\boldsymbol{u}$ and (37) of $\boldsymbol{\theta}$ (see Appendix), one can write:

$$
\begin{aligned}
\boldsymbol{u}(\boldsymbol{y})-\boldsymbol{u}(\boldsymbol{x}) & =\rho \sum_{k=1}^{N} \boldsymbol{u}^{k} \hat{N}^{k}(\rho, \varphi ; \boldsymbol{\eta}) \\
\boldsymbol{B}^{k}(\boldsymbol{y})-\boldsymbol{B}^{k}(\boldsymbol{x}) & =\rho \hat{\boldsymbol{B}}^{k}(\rho, \varphi ; \boldsymbol{\eta})
\end{aligned}
$$

From eqs. (50) to (53), it is easy to see that all singular integrals are recast into regular integrals expressed in terms of $(\rho, \varphi)$. This makes full use of the regularization. The subsequent numerical integrations can be performed with standard product Gaussian quadrature formulas, using a last coordinate change $(\rho, \varphi) \rightarrow\left(v_{1}, v_{2}\right)$ in order to recover an integral over the square $[-1,1]^{2}$.

\section{$6 \quad$ Numerical examples}

Example 1 - Round bar with a penny-shaped axial crack. An internal penny-shaped plane crack of radius $R_{1}$ is situated in a cylindrical bar (length $2 H$, external radius $R>R_{1}$ ); the crack plane is the plane of symmetry orthogonal to the axis of rotational symmetry, as shown on figure 5. The bar is subjected to a uniform tension $p$, along the direction orthogonal to the crack plane (mode $I$ ). An approximate solution for $K_{I}$, obtained using semi-analytical methods, is known for this problem (Tada, Paris \& Irwin [28]) in the form:

$$
K_{I}=\frac{1}{1-\left(R_{1}^{2} / R^{2}\right)} p \sqrt{\pi R_{1}} F\left(R_{1} / R\right)
$$

and values for $F$ are provided by approximate formulas.

Figure 5 should appear here. 
Two different meshes, as shown on figures 6, 7, were used for one-eighth of the structure. The numerical values obtained for $G$ at the crack front nodes are compared with the above reference solution on figures 8,9 , which show the good agreement between them.

Figure 6 should appear here.

Figure 7 should appear here.

Figure 8 should appear here.

Figure 9 should appear here.

Example 2 - Semi-elliptical surface crack. As a second illustrative example, the case of a semi-elliptical surface crack situated in a symmetry plane of a rectangular parallepiped is considered. The geometrical notations are given in figure 10 . The aspect ratio $b / a$ is the ellipticity of the crack ( $b=a$ for the semicircular crack). For comparison with other available solutions, the parallepiped is subjected to a uniform tension $p$, along the direction orthogonal to the crack plane (mode $I$ ). Owing to geometrical symmetry, only one-quarter of the initial parallelipiped is discretized into a total of 136 9-noded boundary elements; the crack front itself is made of 6 element edges, so that $G$ and $\boldsymbol{\theta}$ are interpolated using 13 nodal values. Two variant meshes M1 and M2 were used, both having six bounary elements along the crack front, so that the angular spacing between crack front nodes (using notations of figure 10) is uniform $(\Delta \theta=\pi / 24$, mesh M1, figure 11) or non-uniform $(\Delta \theta=\pi / 32$ (resp. $\pi / 16)$ for $\theta \in[0, \pi / 4]$ (resp. $\theta \in[\pi / 4, \pi / 2])$, mesh M2, figure 12); $\theta=0$ being the angular location of the crack edge.

Figure 10 should appear here.

Figure 11 should appear here.

Figure 12 should appear here.

Numerical values of the non-dimensional SIF $K_{I}^{\star}=K_{I} / K_{I}^{e}$ were obtained from the computed values of $G$ computed with the present method, using:

$$
K_{I}=\left[\frac{2 \mu}{1+\nu} G\right]^{1 / 2} \quad K_{I}^{e}=\frac{1}{Q} p \sqrt{\pi a}
$$

the shape factor $Q$ being defined as:

$$
Q=E(k)=\int_{0}^{\pi / 2} \sqrt{1-k^{2} \sin ^{2} \theta} d \theta \quad k^{2}=\left\{\begin{array}{cc}
1-(b / a)^{2} & (b \leq a) \\
1-(a / b)^{2} & (b \geq a)
\end{array}\right.
$$


They are compared to other numerical results from Newman and Raju [17] (figures 13 to 16) and from Tanaka and Itoh [29] (figures 14, 15). The latter ones were obtained using a sophisticated spcial crack-front element which allows for the modelling of both the square-root crack front singularity and the crack edge singularity (whose exponent differs from $-1 / 2$ except for $\nu=0$ ), and are thus expected to provide the better reference solution near the crack edge. One sees on figures 14, 15 that our results show a better tendency to reproduce the small peak near the crack edge when mesh M2, which is finer than M1 near the crack edge, is used. Generally speaking, our results agree reasonably well with the reference ones. For comparison sake, we have also depicted on figures 13 to 16 the values of $K_{I}^{\star}$ obtained by extrapolation of the crack opening displacement. They tend to be somewhat less good than those obtained using the present approach, dspite the use of quarter-node elements along the crack front.

Figure 13 should appear here.

Figure 14 should appear here.

Figure 15 should appear here.

Figure 16 should appear here.

\section{Conclusion}

In this paper, a novel approach for the computation of energy release rate $G(s)$ has been presented for 3-D elastic fracture. It is based on a boundary-only approach for the formulation of perturbations of the elastic potential energy at equilibrium induced by crack front virtual advances. As such, this work parallels other investigations devoted to the FEM-based ' $\theta$-method'.

A key ingredient in the present approach is the use of the derivative BIE which governs the elastic sensitivities on the boundary. The derivative BIE itself of course is also applicable to other kinds of situations, like shape optimization or inverse problems.

The extra computational cost associated with the building and solution of this derivative BIE is reasonable thanks to the fact that the primary and derivative BIEs share the same governing operator, which is thus build and factored only once. The numerical examples presented show the potential of the method. In particular, they tend to be more accurate than the evaluations based on displacement extrapolation produced by the same run, although quarter-node elements were used along the crack front. 
Further developments should include the study of actual crack growth (including stability and non-bifurcation considerations) under Griffith criterion. This requires that the elastic potential energy be differentiated up to the second order with respect to crack front advances.

\section{References}

[1] Aithal R., Saigal S. - Shape sensitivity analysis in thermal problems using BEM. Eng. Anal. with Boundary El., present issue.

[2] Barone M.R., Yang R.J. - A Boundary Element Approach for Recovery of Shape Sensitivities in Three-dimensional Elastic Solids. Comp. Meth. in Appl. Mech. Engng. 74, 69-82, 1989.

[3] Bonnet M. - BIE and material differentiation applied to the formulation of obstacle inverse problems. Eng. Anal. with Boundary El., present issue.

[4] Bonnet M. - Regularized BIE formulations for first- and second-order shape sensitivity of elastic fields. Contribution to a special issue of Computers and Structures, (S. Saigal, guest editor.), to appear, 1994.

[5] Bui H.D. - Mécanique de la rupture fragile. Masson, 1978.

[6] Bui H.D., Loret B., Bonnet M. - Régularisation des équations intégrales de l'élastostatique et de l'élastodynamique. C.R. Acad. Sci. Paris, série II 300, 633-636, 1985.

[7] Chor J.O., Kwak B.M. - Boundary integral equation method for shape optimization of elastic structures. Int. J. Num. Meth. in Eng,. 26, 1579-1595, 1988.

[8] Cruse T.A. - Boundary Element Analysis in Computational Fracture Mechanics. Kluwer Academic Publishers, 1988.

[9] Delorenzi H.G. - On the energy release rate and the $J$-integral for 3 -D crack configurations. Int. J. Fract. 19, 183-194, 1982.

[10] Destuynder Ph., Djaoua M., Lescure S. - Quelques remarques sur la mécanique de la rupture élastique. J. Mécan. Théor. Appl. 2, 113-135, 1983.

[11] Haug, Choi, Komkov - Design Sensitivity Analysis of Structural Systems, Academic Press, 1986. 
[12] Hellen T.K. - On the method of virtual crack extension. Int. J. Num. Meth. in Eng,. 9, 187-207, 1975.

[13] Kupradze V.D. (Editor) - Three-dimensional problems of the mathematical theory of elasticity and thermoelasticity. North Holland, 1979.

[14] MERIC R.A. - Differential and integral sensitivity formulations and shape optimization by BEM. Eng. Anal. with Boundary El., present issue.

[15] Mialon P. - Calcul de la dérivée d'une grandeur par rapport à un fond de fissure par la mèthode $\theta$. Bulletin EDF/DER série C vol.3, 1987.

[16] Mukherjee S., Chandra A. - A boundary element formulation for design sensitivities in problems involving both geometric and material nonlinearities. Mathl. Comput. Modelling. 15, 245-255, 1991.

[17] Newman J.C., RaJu I.S. - An empirical stress-intensity factor equation for the surface crack. Engng. Frac. Mech. 15, 185-192, 1981.

[18] Nguyen Q.S., Stolz C., Debruyne G. - Energy methods in fracture mechanics: stability, bifurcation and second variations. Eur. J. Mech. A/Solids 9, 157-173, 1990.

[19] NGuyen Q.S. - Bifurcation and stability in dissipative media (plasticity, friction, fracture). Appl. Mech. Rev. 37, 1-31, 1994.

[20] Ohtsuka A. - Generalized J-integral and three-dimensional fracture mechanics. $H i$ roshima Math. J. 11, 21-52, 1981.

[21] PARKs D.M. - A stiffness derivative finite element technique for determination of crack tip strees intensity factors. Int. J. Fract. 10, 487-501, 1974.

[22] Petryk H., Mroz Z. - Time derivatives of integrals and functionals defined on varying volume and surface domains. Arch. Mech. 38, 694-724, 1986.

[23] Pradeilles-Duval R.M. - Evolution de systèmes avec surfaces de discontinuité mobiles: application au délaminage. Thèse de Doctorat de l'Ecole Polytechnique, décembre 1992.

[24] Rizzo F.J., Shippy D.J., Rezayat M. - A boundary integral equation method for radiation and scattering of elastic waves in three-dimensions. Int. J. Num. Meth. in Eng,. 23, 425-436, 1986 . 
[25] Rizzo F.J., Shippy D.J. - An advanced boundary integral equation method for threedimensional elasticity. Int. J. Num. Meth. in Eng,. 11, 1753-1768, 1977.

[26] Salençon J. - Mécanique des milieux continus. Presse de l'Ecole Polytechnique, 1992

[27] Suo X.Z., Combescure A. - Sur une formulation mathématique de la dérivée de l'énergie potentielle en théorie de la rupture fragile. C.R. Acad. Sci. Paris, série II 308, 1119-1122, 1989.

[28] TADA, PARIS \& IRWIN - The stress analysis of crack handbook. Del. Research Corporation, Hellertown, Pennsylvania, USA (1973).

[29] TAnaka M., Iтон H. - New crack elements for boundary element analysis of elastostatics considering arbitrary stress singularities. Appl. Math. Modelling 11, 357-363, $198 \%$.

[30] Tikhonov A.N., Arsenin V.Y. - Solutions to ill-posed problems. Winston-Wiley, New York, 1977.

[31] Wadier Y., Malak O. - The theta method applied to the analysis of 3D-elastic-plastic cracked bodies. SMIRT, Los Angeles 1989.

[32] Zhang Q., MukherJee S. - Second-order design sensitivity analysis for linear elastic problems by the derivative boundary element method. Comp. Meth. in Appl. Mech. Engng. 86, 321-335, 1991.

\section{A Material derivative of $\mathrm{d} S$ and $n$}

For the sake of completeness, eq. (7) is briefly established. First the material derivative of a material vector $\boldsymbol{a}$ attached to the moving point $\boldsymbol{y}=\boldsymbol{\Phi}(\boldsymbol{Y} ; p)$ is given by:

$$
\stackrel{\star}{a}=\nabla \theta \cdot a
$$

Then, let $S_{p}$ be a material surface, and denote by $(\boldsymbol{a}, \boldsymbol{b})$ a pair of material vectors attached to a material point $\boldsymbol{y}=\boldsymbol{\Phi}(\boldsymbol{Y} ; p)$, chosen so as to be unitary and orthogonal at a fixed value $p_{0}$ of $p$ and to belong to the tangent plane at $\boldsymbol{y}$ to $S_{p}$ for all $p$ in a neighbourhood of $p_{0}$. For any such $p$, the unit normal to $S_{p}$ at $\boldsymbol{y}$ is thus given by

$$
\boldsymbol{n}(\boldsymbol{y} ; p)=\frac{1}{|\boldsymbol{a} \wedge \boldsymbol{b}|}(\boldsymbol{a} \wedge \boldsymbol{b})
$$


Moreover, the surface differential element $\mathrm{d} S$ at $\boldsymbol{y}$ is proportional to $|\boldsymbol{a} \wedge \boldsymbol{b}|$, so that:

$$
\stackrel{\star}{\mathrm{d} S}=|\boldsymbol{a} \wedge \boldsymbol{b}|^{\star} \mathrm{d} S
$$

The material derivative of the vector product $\boldsymbol{a} \wedge \boldsymbol{b}$ is then taken for the particular value $p_{0}$ of $p$, using (54):

$$
\begin{aligned}
(\boldsymbol{a} \wedge \boldsymbol{b})^{\star} & =(\boldsymbol{\nabla} \boldsymbol{\theta} . \boldsymbol{a}) \wedge \boldsymbol{b}+\boldsymbol{a} \wedge(\boldsymbol{\nabla} \boldsymbol{\theta} \cdot \boldsymbol{b}) \\
& =\left[(\boldsymbol{\nabla} \boldsymbol{\theta})_{a a}+(\boldsymbol{\nabla} \boldsymbol{\theta})_{b b}\right] \boldsymbol{n}-(\boldsymbol{\nabla} \boldsymbol{\theta})_{n a} \boldsymbol{a}-(\boldsymbol{\nabla} \boldsymbol{\theta})_{n b} \boldsymbol{b} \\
& =\left(\operatorname{div}_{S} \boldsymbol{\theta}\right) \boldsymbol{n}-\boldsymbol{n} \cdot \boldsymbol{\nabla}_{S} \boldsymbol{\theta}
\end{aligned}
$$

where the fact that $(\boldsymbol{a}, \boldsymbol{b}, \boldsymbol{n})$ is an orthonormal vector frame at $p=p_{0}$ has been used. Moreover, one has:

$$
|\boldsymbol{a} \wedge \boldsymbol{b}|^{\star}=\frac{\boldsymbol{a} \wedge \boldsymbol{b}}{|\boldsymbol{a} \wedge \boldsymbol{b}|} \cdot(\boldsymbol{a} \wedge \boldsymbol{b})^{\star}=\boldsymbol{n} \cdot(\boldsymbol{a} \wedge \boldsymbol{b})^{\star}=\operatorname{div}_{S} \boldsymbol{\theta}
$$

Eq. (7) is then easily obtained from (55), (56), (57), (58).

\section{B Modified shape functions for singular integration}

Shape functions for 9-noded quadrilateral element. The classical Lagrangian shape functions for the 9-noded quadrilateral element are:

$$
\begin{array}{lll}
N_{1}(\boldsymbol{\xi})=S_{1}\left(\xi_{1}\right) S_{1}\left(\xi_{2}\right) & N_{4}(\boldsymbol{\xi})=S_{3}\left(\xi_{1}\right) S_{2}\left(\xi_{2}\right) & N_{7}(\boldsymbol{\xi})=S_{1}\left(\xi_{1}\right) S_{3}\left(\xi_{2}\right) \\
N_{2}(\boldsymbol{\xi})=S_{2}\left(\xi_{1}\right) S_{1}\left(\xi_{2}\right) & N_{5}(\boldsymbol{\xi})=S_{3}\left(\xi_{1}\right) S_{3}\left(\xi_{2}\right) & N_{8}(\boldsymbol{\xi})=S_{1}\left(\xi_{1}\right) S_{2}\left(\xi_{2}\right) \\
N_{3}(\boldsymbol{\xi})=S_{3}\left(\xi_{1}\right) S_{1}\left(\xi_{2}\right) & N_{6}(\boldsymbol{\xi})=S_{2}\left(\xi_{1}\right) S_{3}\left(\xi_{2}\right) & N_{9}(\boldsymbol{\xi})=S_{2}\left(\xi_{1}\right) S_{2}\left(\xi_{2}\right)
\end{array}
$$

with the $S_{i}$ given by (41). Their derivatives $N_{, \alpha}$ are thus given by

$$
N_{, \alpha}(\boldsymbol{\xi})=S_{i}^{\prime}\left(\xi_{1}\right) S_{j}\left(\xi_{2}\right)+S_{i}\left(\xi_{1}\right) S_{j}^{\prime}\left(\xi_{2}\right)
$$

with appropriately chosen $i, j$.

Modified shape functions. Singular integrations use modified shape functions $\hat{N}$ such that:

$$
N(\boldsymbol{\xi})-N(\boldsymbol{\eta})=\rho \hat{N}(\rho, \varphi ; \boldsymbol{\eta})
$$

First, note that, for any pair $f(\boldsymbol{\xi}), g(\boldsymbol{\xi})$ of regular functions:

$$
\widehat{f g}(\rho, \varphi ; \boldsymbol{\eta})=\hat{f}(\rho, \varphi ; \boldsymbol{\eta}) g(\boldsymbol{\xi})+f(\boldsymbol{\eta}) \hat{g}(\rho, \varphi ; \boldsymbol{\eta})
$$


so that the $\hat{N}, \hat{N}_{, \alpha}$, eqs. (59), (60) can be readily obtained in terms of the $\hat{S}_{i}, \hat{S}^{\prime}{ }_{i}$, with:

$$
\begin{array}{ll}
\hat{S}_{1}\left(\rho, \varphi ; \eta_{\alpha}\right)=c_{\alpha}\left[2 \eta_{\alpha}-1+\rho c_{\alpha}\right] / 2 & \hat{S}^{\prime}{ }_{1}\left(\rho, \varphi ; \eta_{\alpha}\right)=c_{\alpha} \\
\hat{S}_{1}\left(\rho, \varphi ; \eta_{\alpha}\right)=c_{\alpha}\left[-2 \eta_{\alpha}-\rho c_{\alpha}\right] & \hat{S}^{\prime}{ }_{1}\left(\rho, \varphi ; \eta_{\alpha}\right)=-2 c_{\alpha} \\
\hat{S}_{3}\left(\rho, \varphi ; \eta_{\alpha}\right)=c_{\alpha}\left[2 \eta_{\alpha}+1+\rho c_{\alpha}\right] / 2 & \hat{S}^{\prime}{ }_{1}\left(\rho, \varphi ; \eta_{\alpha}\right)=c_{\alpha}
\end{array}
$$

In the above formula, $c_{1}, c_{2}$ stand for $\cos \varphi, \sin \varphi$ respectively.

Modified vector shape function $\hat{B}_{j}^{m}$. It is defined by:

$$
\boldsymbol{B}^{m}(\boldsymbol{\xi})-\boldsymbol{B}^{m}(\boldsymbol{\eta})=\rho \hat{\boldsymbol{B}}^{m}(\rho, \varphi ; \boldsymbol{\eta})
$$

From the definition (40) and using (61), $\hat{\boldsymbol{B}}^{m}$ is given by:

$$
\hat{\boldsymbol{B}}^{m}(\rho, \varphi, \boldsymbol{\eta})=\left(\hat{f}\left(\rho, \varphi ; \eta_{2}\right) S_{m}\left(\xi_{1}\right)+f\left(\eta_{1}\right) \hat{S}_{m}\left(\rho, \varphi ; \eta_{1}\right)\right) \boldsymbol{\nu}(\boldsymbol{\xi})+f\left(\eta_{2}\right) S_{m}\left(\eta_{1}\right) \hat{\boldsymbol{\nu}}_{m}(\rho, \varphi, \boldsymbol{\eta})
$$

Next, expression (39) for $\boldsymbol{\nu}$ and the rule (61) give:

$$
\begin{aligned}
\hat{\boldsymbol{\nu}}_{m}(\rho, \varphi, \boldsymbol{\eta})= & \frac{g_{1 \alpha}}{\sqrt{g_{11} J}}(\boldsymbol{\xi}) \hat{\boldsymbol{a}}_{1}(\rho, \varphi ; \boldsymbol{\eta})-\frac{g_{1 \alpha}}{\sqrt{g_{11} J}}(\boldsymbol{\xi}) \hat{\boldsymbol{a}}_{2}(\rho, \varphi ; \boldsymbol{\eta}) \\
& +\left(\frac{g_{12}}{\sqrt{g_{11} J}}\right)^{\wedge}(\rho, \varphi ; \boldsymbol{\eta}) \boldsymbol{a}_{1}(\boldsymbol{\eta})-\left(\frac{g_{11}}{\sqrt{g_{11} J}}\right)^{\wedge}(\rho, \varphi ; \boldsymbol{\eta}) \boldsymbol{a}_{2}(\boldsymbol{\eta})
\end{aligned}
$$

with:

$$
\hat{\boldsymbol{a}}_{\alpha}(\rho, \varphi ; \boldsymbol{\eta})=\sum_{k=1}^{\mathrm{NU}} \hat{N}_{k, \alpha}(\rho, \varphi ; \boldsymbol{\eta}) \boldsymbol{y}^{k}
$$

Finally, one can show that:

$$
\left(\frac{g_{1 \alpha}}{\sqrt{g_{11} J}}\right)^{\wedge}(\rho, \varphi ; \boldsymbol{\eta})=\frac{1}{D_{\alpha}}\left(A_{11}^{\alpha} \hat{g}_{11}+A_{12}^{\alpha} \hat{g}_{12}+A_{22}^{\alpha} \hat{g}_{22}\right) \quad(\alpha=1,2)
$$

with

$$
\begin{aligned}
\hat{g}_{\alpha \beta}(\rho, \varphi ; \boldsymbol{\eta}) & =\hat{\boldsymbol{a}}_{\alpha}(\rho, \varphi ; \boldsymbol{\eta}) \cdot \boldsymbol{a}_{\beta}(\boldsymbol{\xi})+\boldsymbol{a}_{\alpha}(\boldsymbol{\eta}) \hat{\boldsymbol{a}}_{\beta}(\rho, \varphi ; \boldsymbol{\eta}) \\
D_{\alpha}(\boldsymbol{\xi}, \boldsymbol{\eta}) & =\sqrt{g_{11}(\boldsymbol{\xi}) J(\boldsymbol{\xi})} \sqrt{g_{11}(\boldsymbol{\eta}) J(\boldsymbol{\eta})}\left[g_{1 \alpha}(\boldsymbol{\xi}) \sqrt{g_{11}(\boldsymbol{\eta}) J(\boldsymbol{\eta})}+g_{1 \alpha}(\boldsymbol{\eta}) \sqrt{g_{11}(\boldsymbol{\xi}) J(\boldsymbol{\xi})}\right] \\
A_{11}^{\alpha}(\boldsymbol{\xi}, \boldsymbol{\eta}) & =g_{1 \alpha}^{2}(\boldsymbol{\eta}) g_{12}^{2}(\boldsymbol{\xi})-g_{\alpha \alpha}(\boldsymbol{\eta}) g_{12}(\boldsymbol{\eta})\left[g_{12}(\boldsymbol{\xi}) g_{11}(\boldsymbol{\eta})+g_{12}(\boldsymbol{\eta}) g_{11}(\boldsymbol{\xi})\right] \\
A_{12}^{\alpha}(\boldsymbol{\xi}, \boldsymbol{\eta}) & =g_{\alpha \alpha}(\boldsymbol{\eta}) g_{11}(\boldsymbol{\eta})\left[g_{12}(\boldsymbol{\xi}) g_{11}(\boldsymbol{\eta})+g_{12}(\boldsymbol{\eta}) g_{11}(\boldsymbol{\xi})\right] \\
A_{22}^{\alpha}(\boldsymbol{\xi}, \boldsymbol{\eta}) & =-g_{1 \alpha}^{2}(\boldsymbol{\eta}) g_{11}^{2}(\boldsymbol{\xi})
\end{aligned}
$$

\section{Expression of $D_{r} B_{r}^{m}$}

One can show using classical differential geometry that, for any vector $\boldsymbol{B}$ such that $\boldsymbol{B} . \boldsymbol{n}=0$ :

$$
\operatorname{div}_{S} \boldsymbol{B} \mathrm{d} S_{y}=\left(\sqrt{J} B^{\alpha}\right)_{, \alpha} \mathrm{d} \xi
$$


Then, from the definition (40) of $\boldsymbol{B}^{m}$, one has:

$$
\operatorname{div}_{S} \boldsymbol{B}^{m} \mathrm{~d} S_{y}=\left\{f S_{m}\left[\left(\sqrt{g_{11}}\right)_{, 2}-\left(\sqrt{\frac{g_{12}}{g_{11}}}\right)_{, 1}\right]+f^{\prime} S_{m} \sqrt{g_{11}}-f S_{m}^{\prime} \sqrt{\frac{g_{12}}{g_{11}}}\right\} \mathrm{d} \xi
$$

The above formulas use the second derivatives $N_{k, \alpha \beta}$ of the shape functions $N_{k}$. 


\section{List of Figures}

1 Multiregion modelling of cracked solids: notation. . . . . . . . . . . . . 23

2 Crack surface $($ shaded area: $E(\partial \Gamma)) \ldots \ldots \ldots \ldots \ldots \ldots$

3 Geometrical support and nodal values for the discretized crack extension velocity 25

4 The vector interpolation function $\boldsymbol{B}^{2} \ldots \ldots \ldots \ldots \ldots$

5 Example 1: Geometrical notations . . . . . . . . . . . . . 27

$6 \quad$ Example 1: Boundary element subdivision of crack plane (coarse mesh) . . . . 28

7 Example 1: Boundary element subdivision of crack plane (fine mesh) . . . . . . . 29

8 Example 1: comparison between numerical results and reference solution (coarse

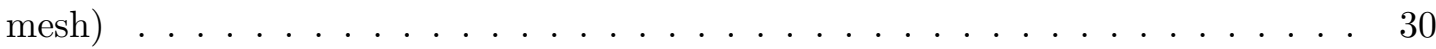

9 Example 1: comparison between numerical results and reference solution (fine

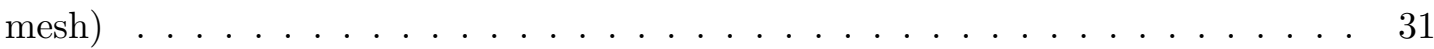

10 Example 2: Geometrical notation for the crack plane . . . . . . . . . . . . 32

11 Example 2: Boundary element subdivision of crack plane (mesh M1, $b=a$ ) . . 33

12 Example 2: Boundary element subdivision of crack plane (mesh M2, $b=0.6 a$ ) . . 34

13 Example 2: comparison between results (shallow semielliptical surface crack, $b=$

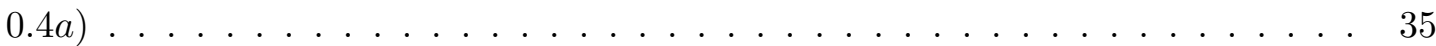

14 Example 2: comparison between results (shallow semielliptical surface crack, $b=$

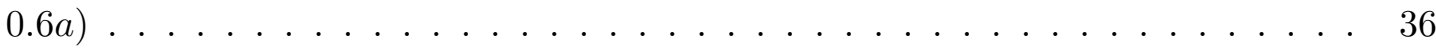

15 Example 2: comparison between results (semicircular surface crack, $b=a$ ) . . . 37

16 Example 2: comparison between results (deep semielliptical surface crack, $b=2 a$ ) 38 


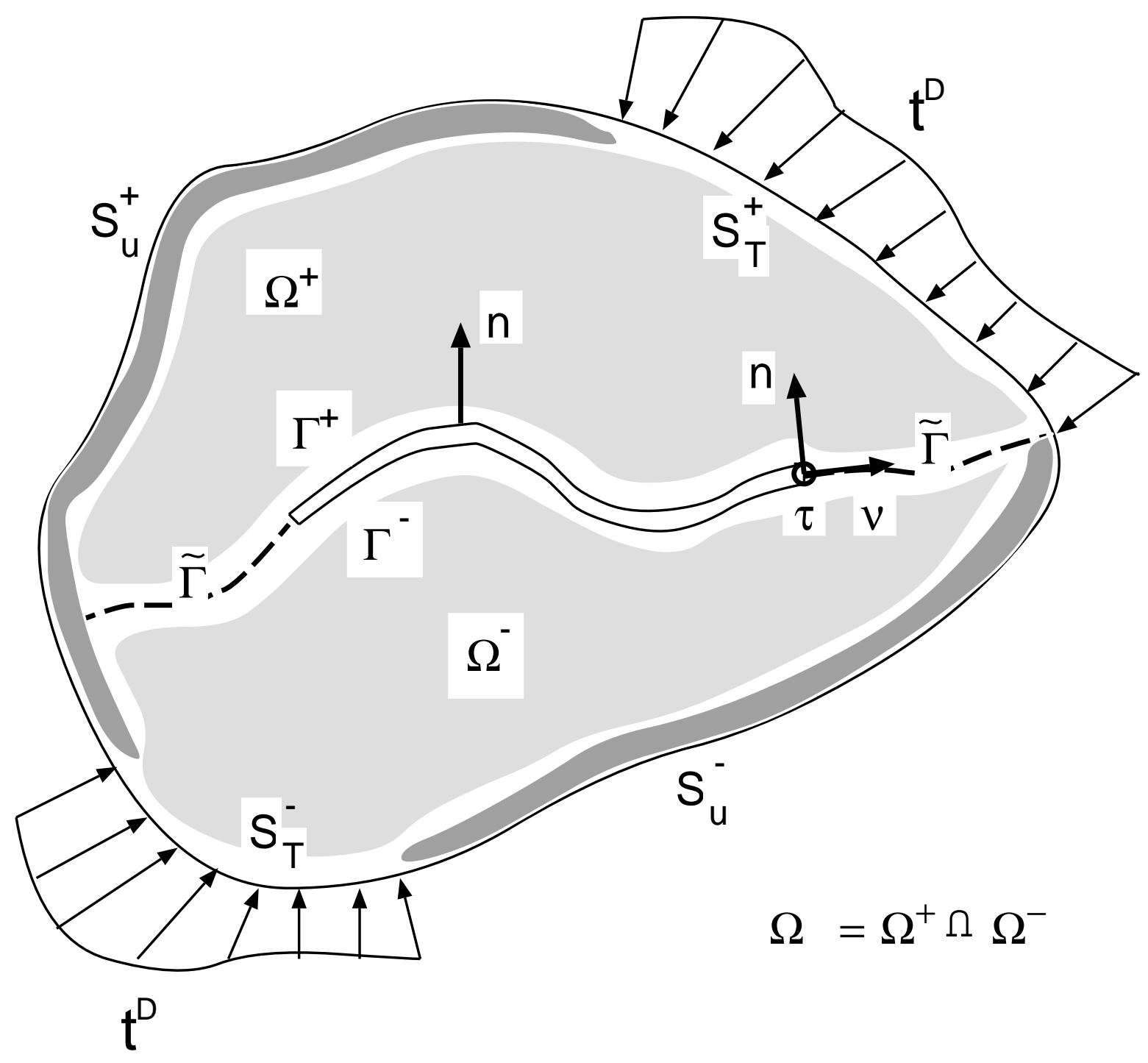

Figure 1: Multiregion modelling of cracked solids: notation. 
- Crack front nodes

- Other nodes

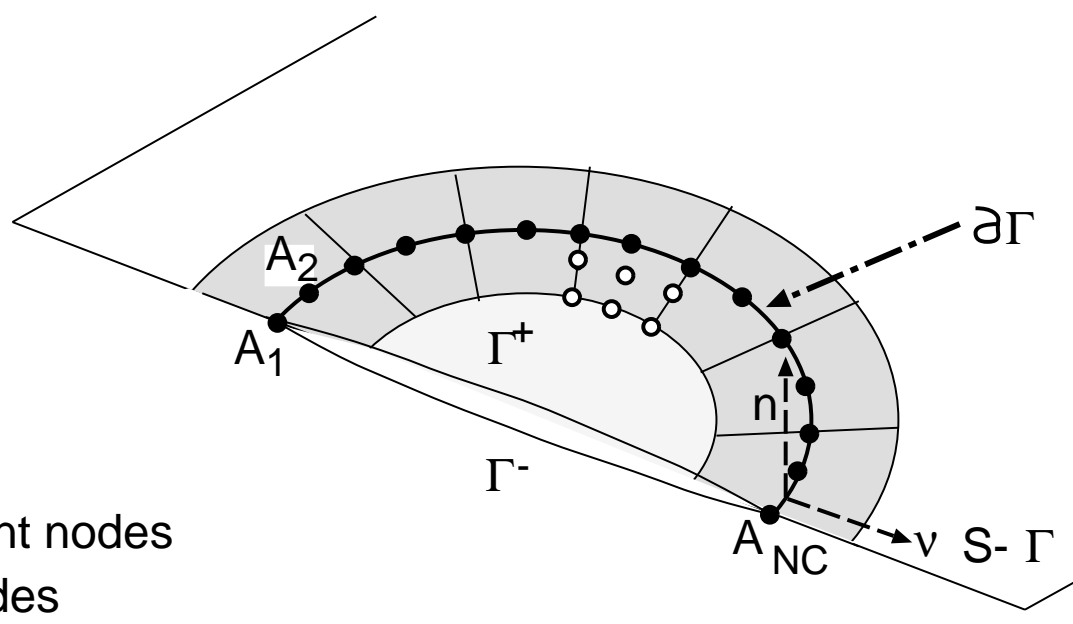

Figure 2: Crack surface (shaded area: $E(\partial \Gamma)$ ) 


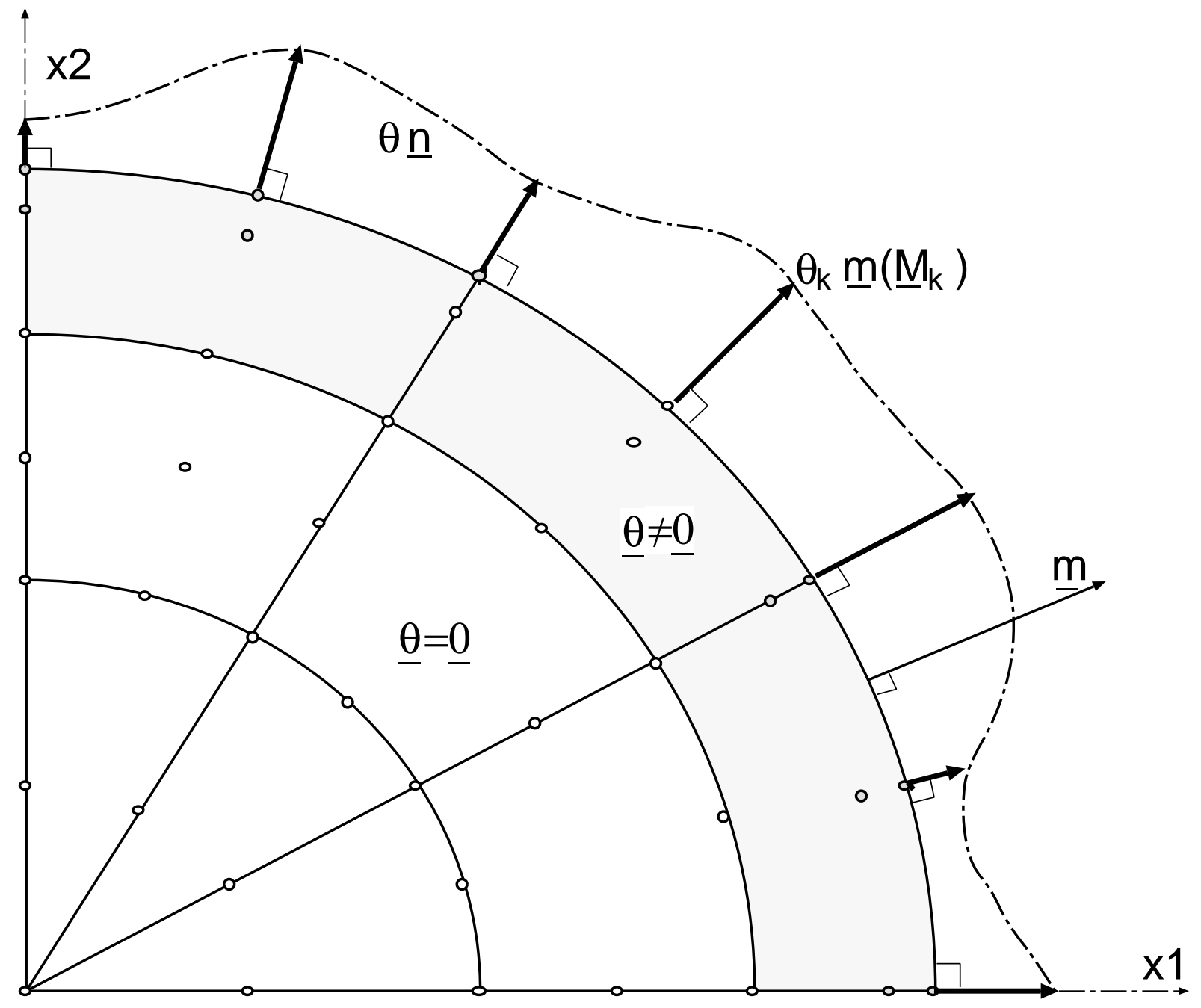

Figure 3: Geometrical support and nodal values for the discretized crack extension velocity 


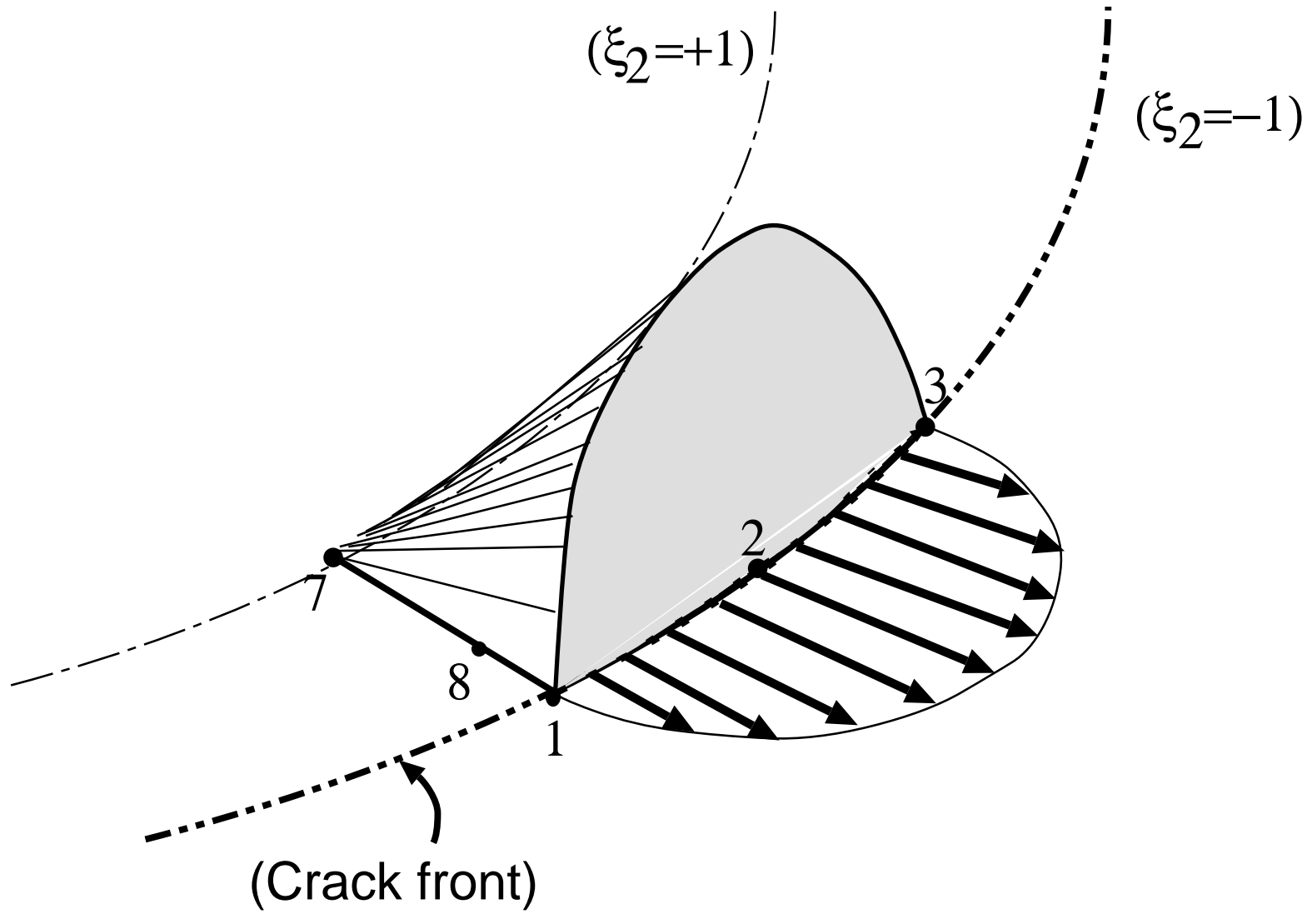

Figure 4: The vector interpolation function $\boldsymbol{B}^{2}$ 


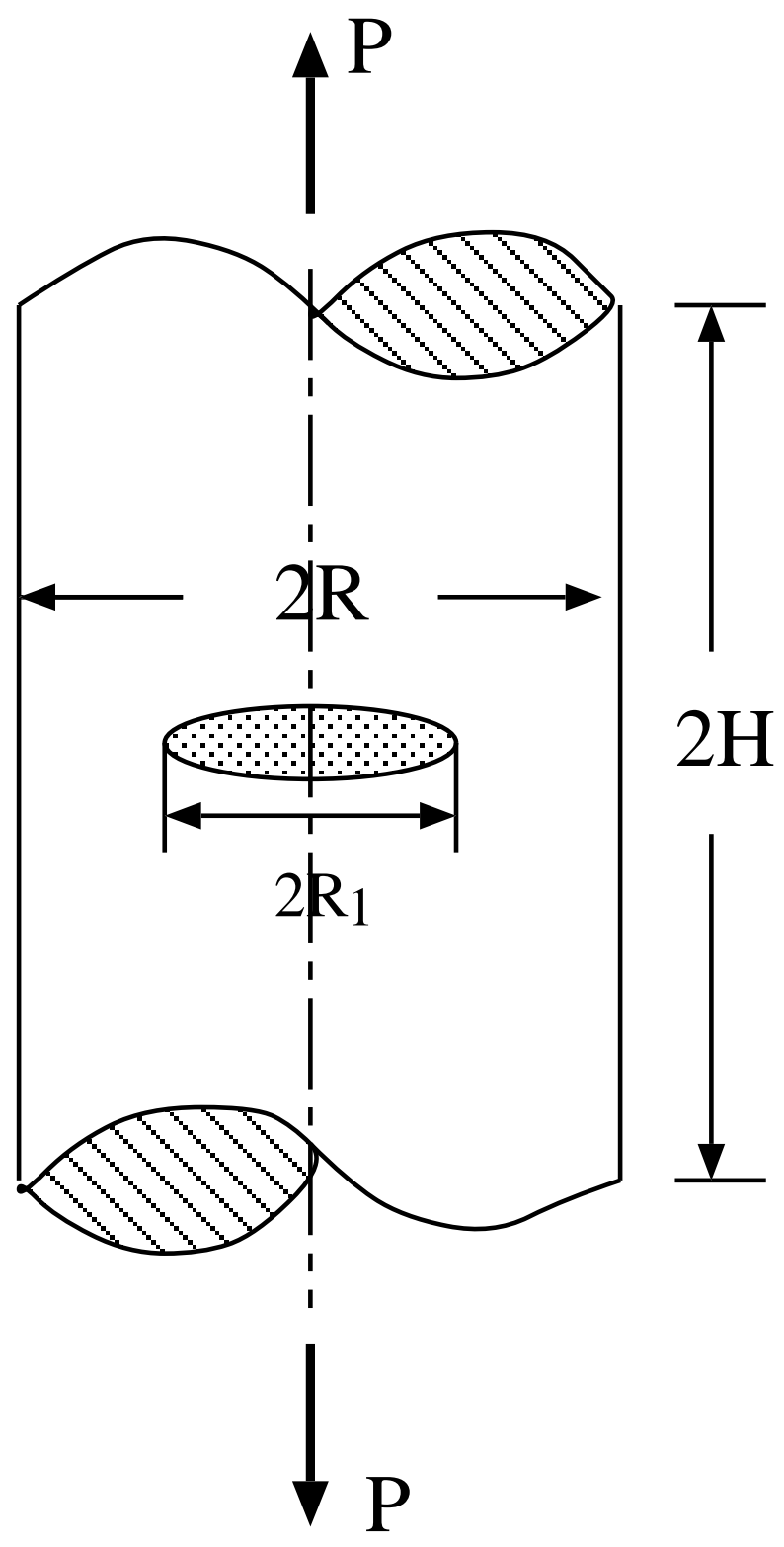

Figure 5: Example 1: Geometrical notations 


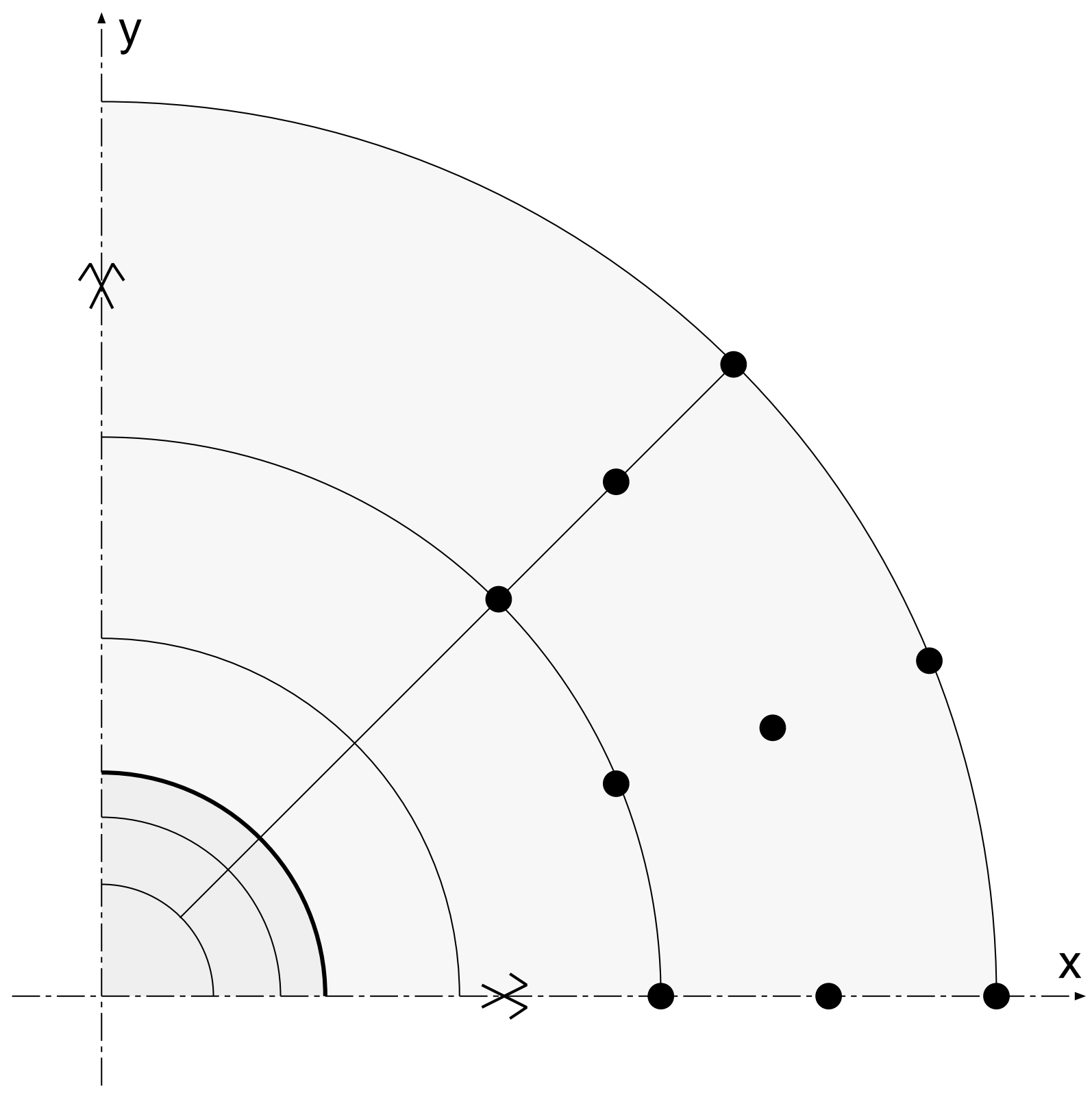

Figure 6: Example 1: Boundary element subdivision of crack plane (coarse mesh) 


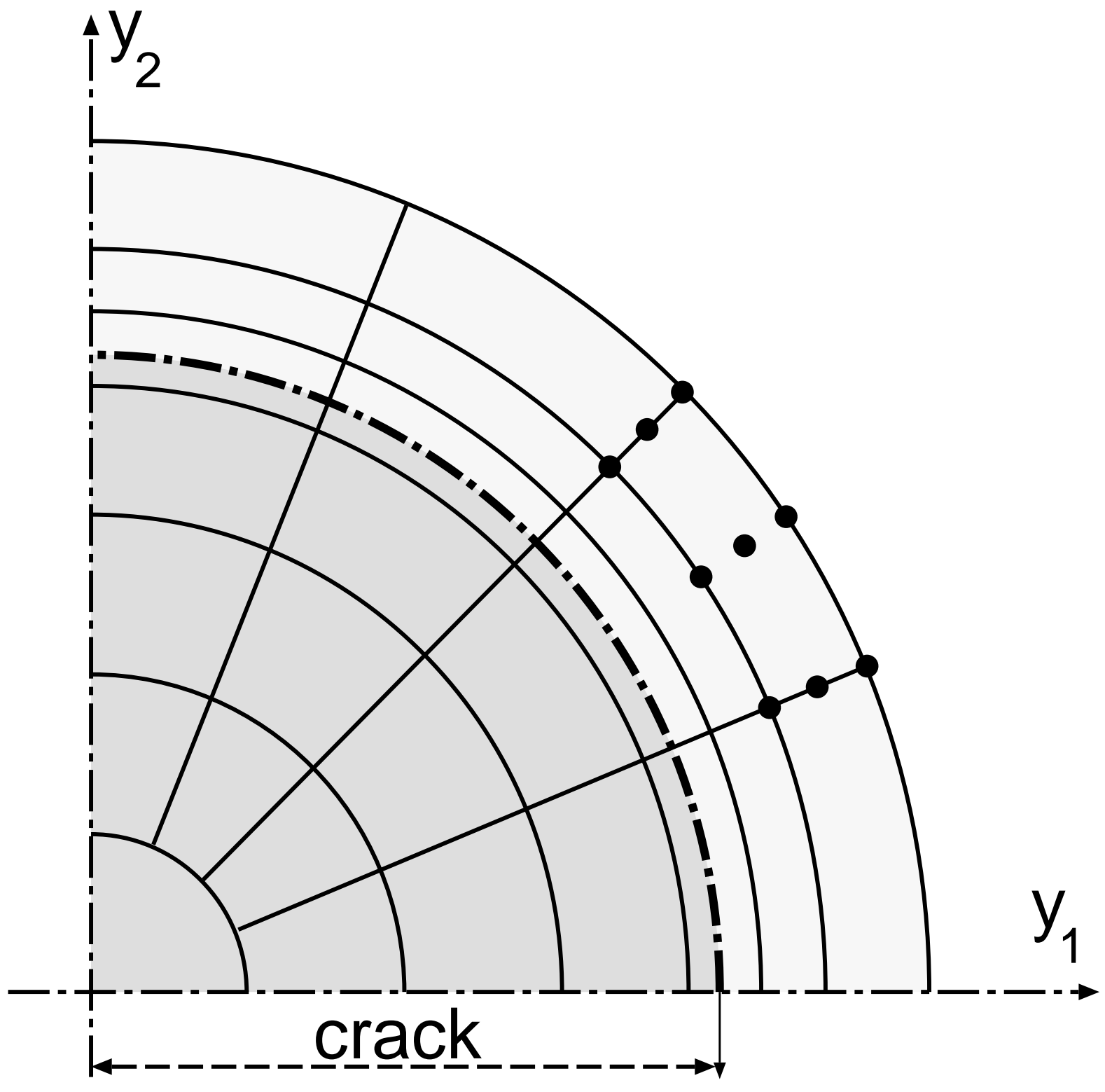

Figure 7: Example 1: Boundary element subdivision of crack plane (fine mesh) 


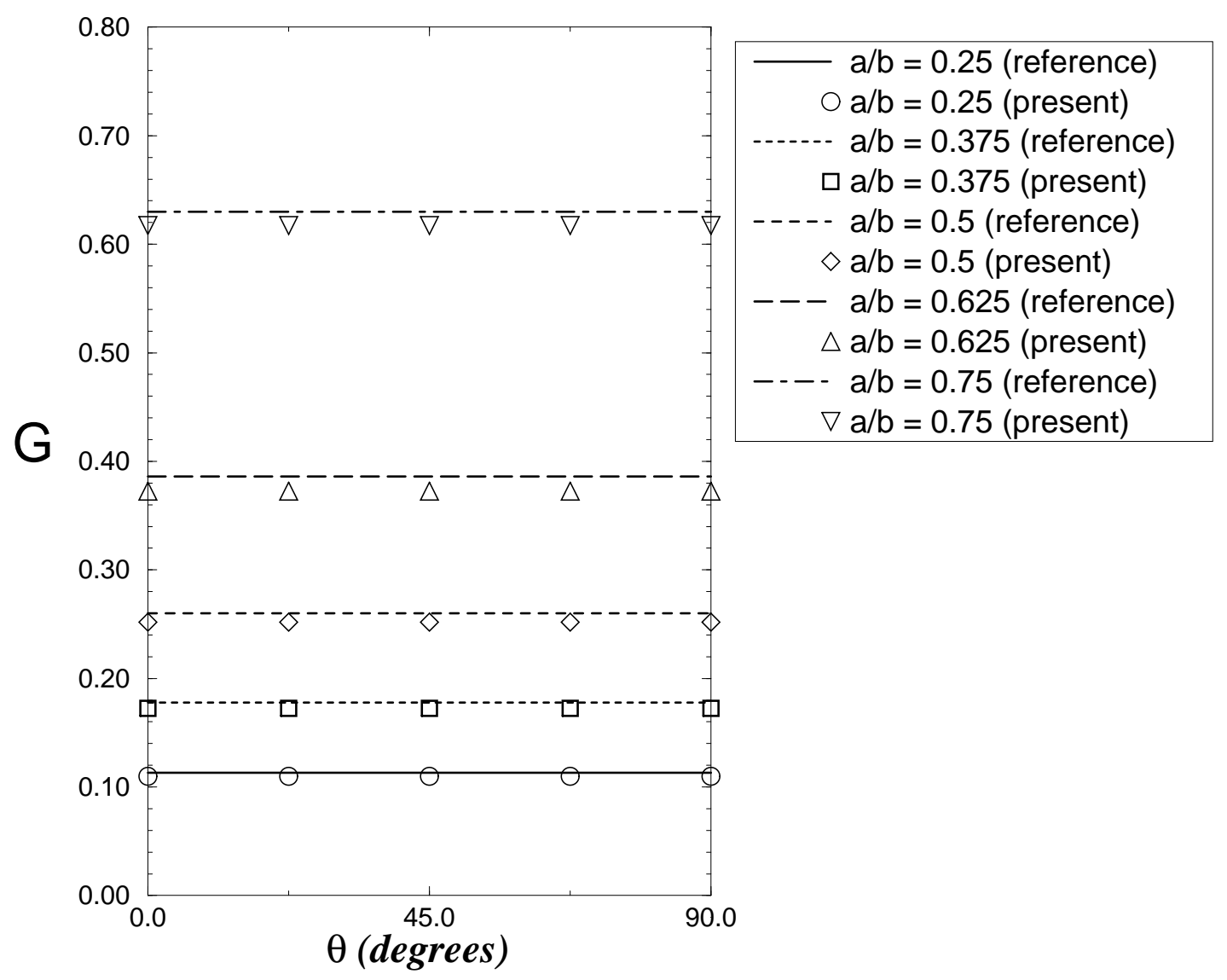

Figure 8: Example 1: comparison between numerical results and reference solution (coarse mesh) 


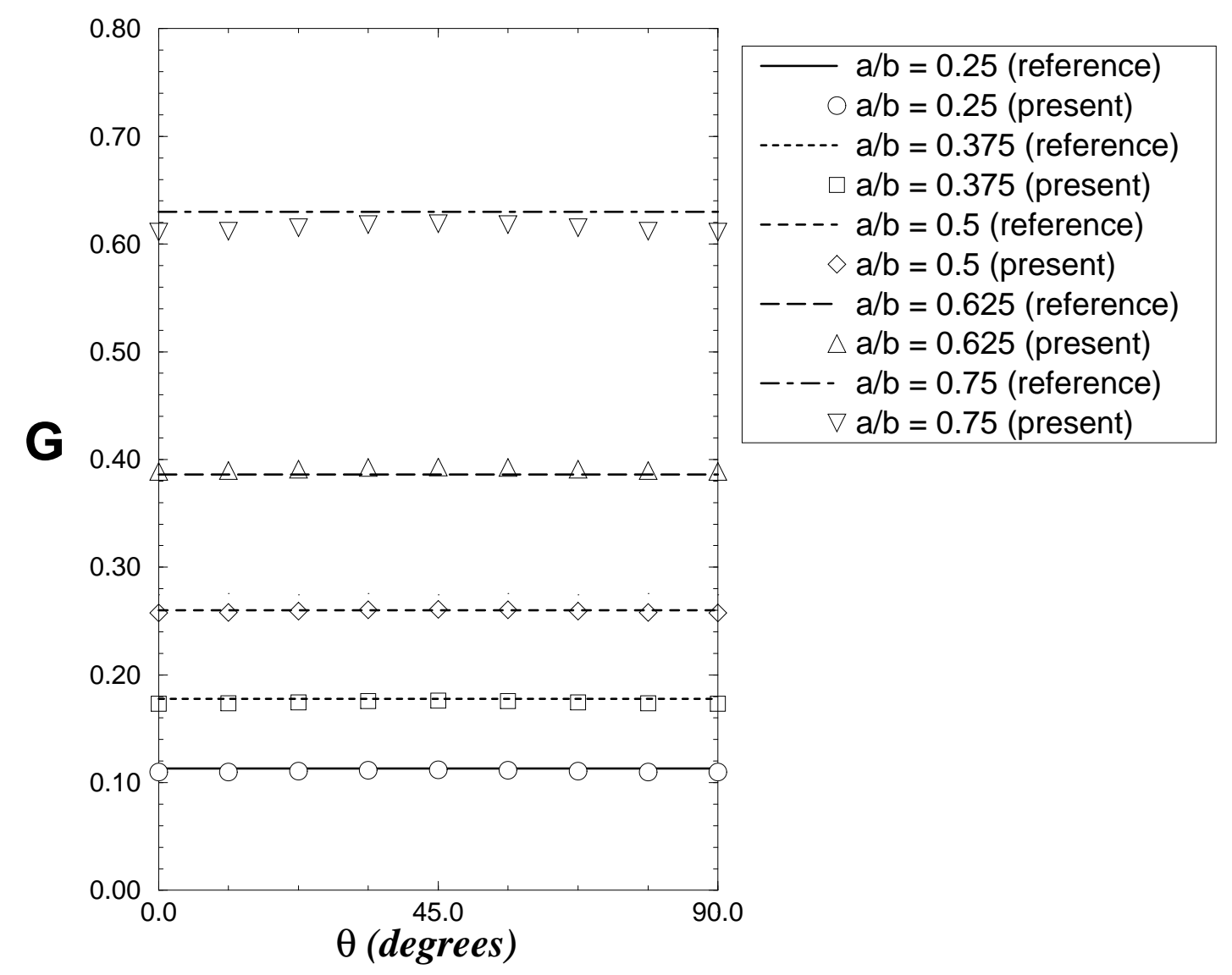

Figure 9: Example 1: comparison between numerical results and reference solution (fine mesh) 


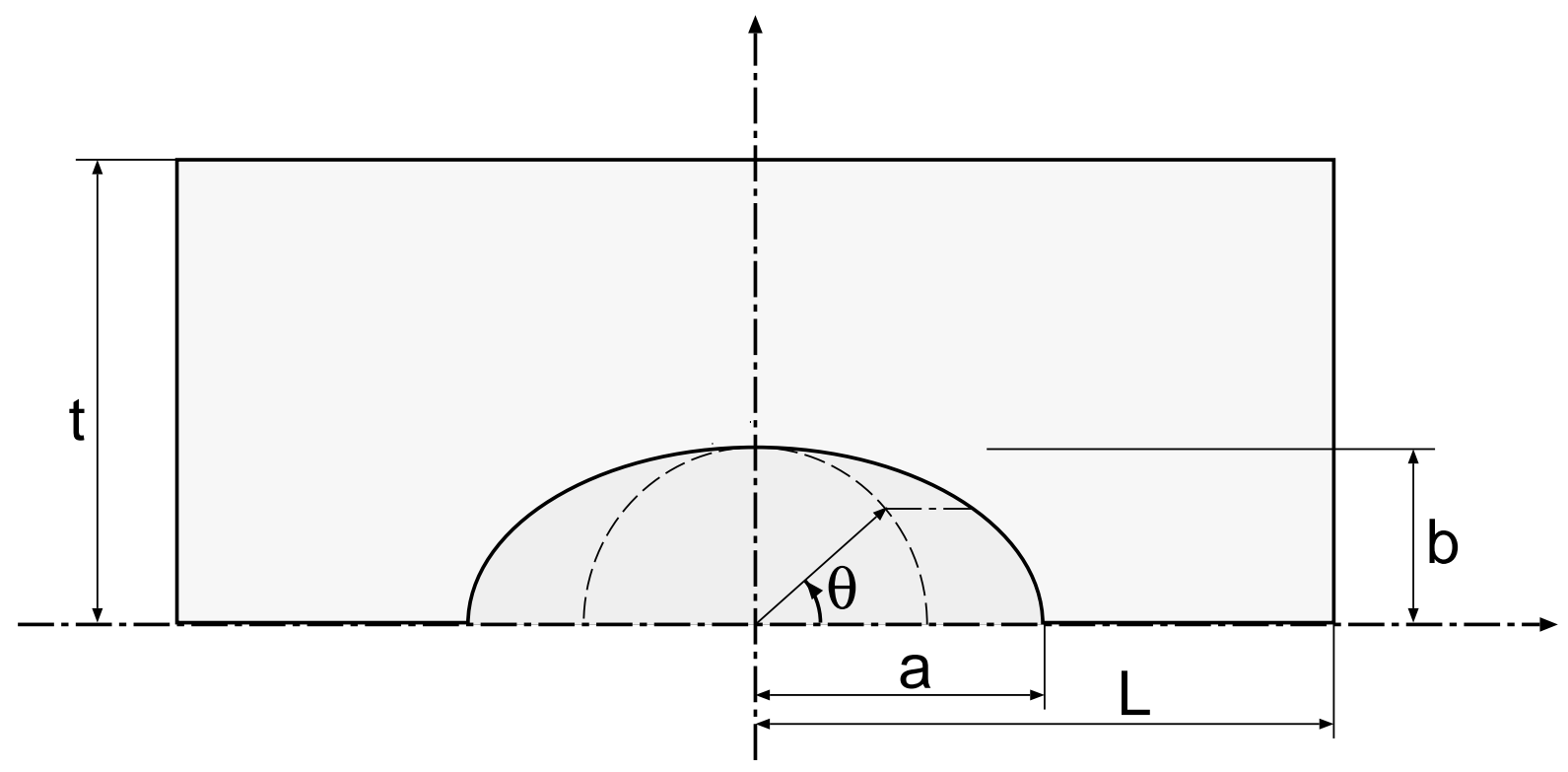

Figure 10: Example 2: Geometrical notation for the crack plane 


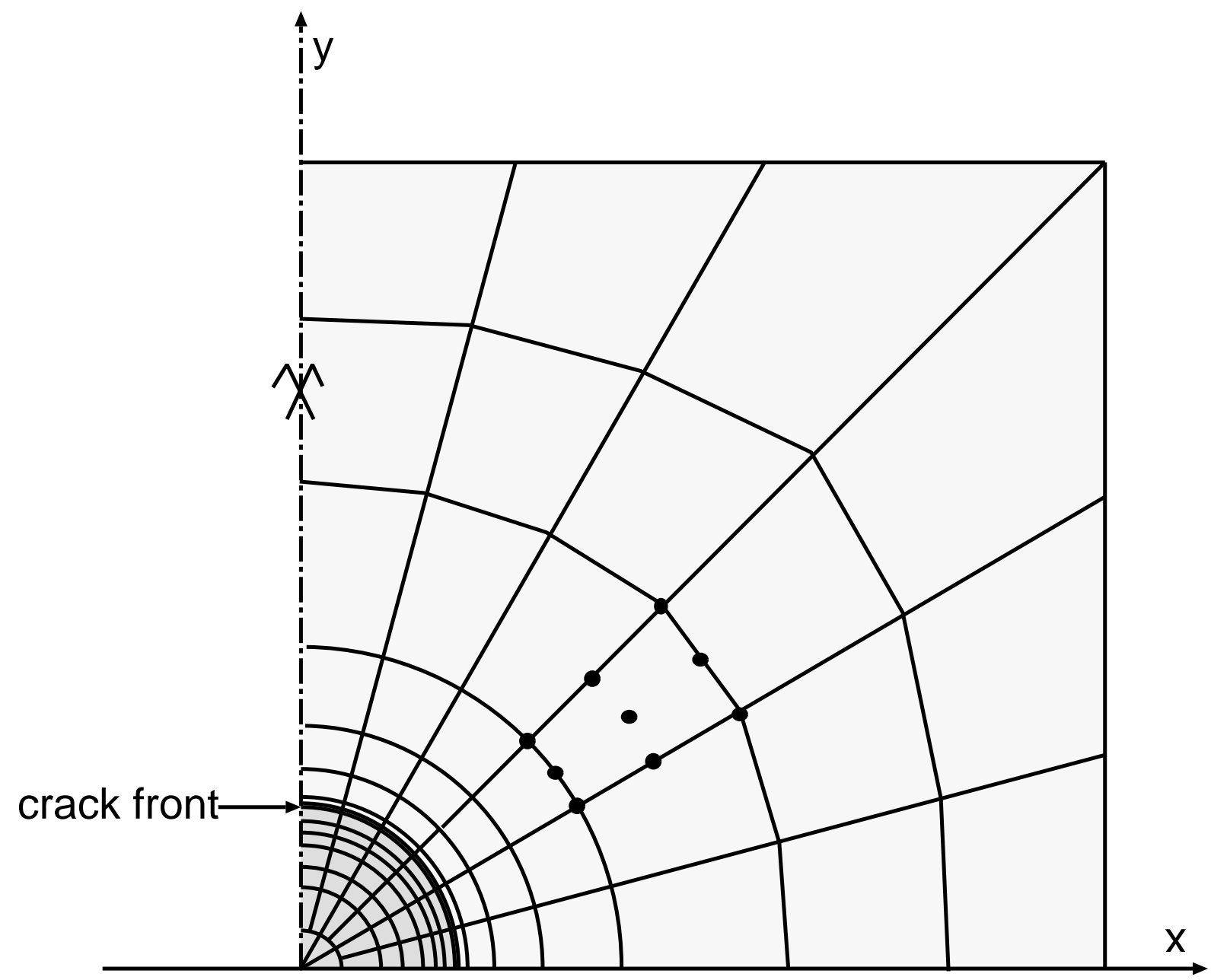

Figure 11: Example 2: Boundary element subdivision of crack plane (mesh $\mathrm{M} 1, b=a)$ 


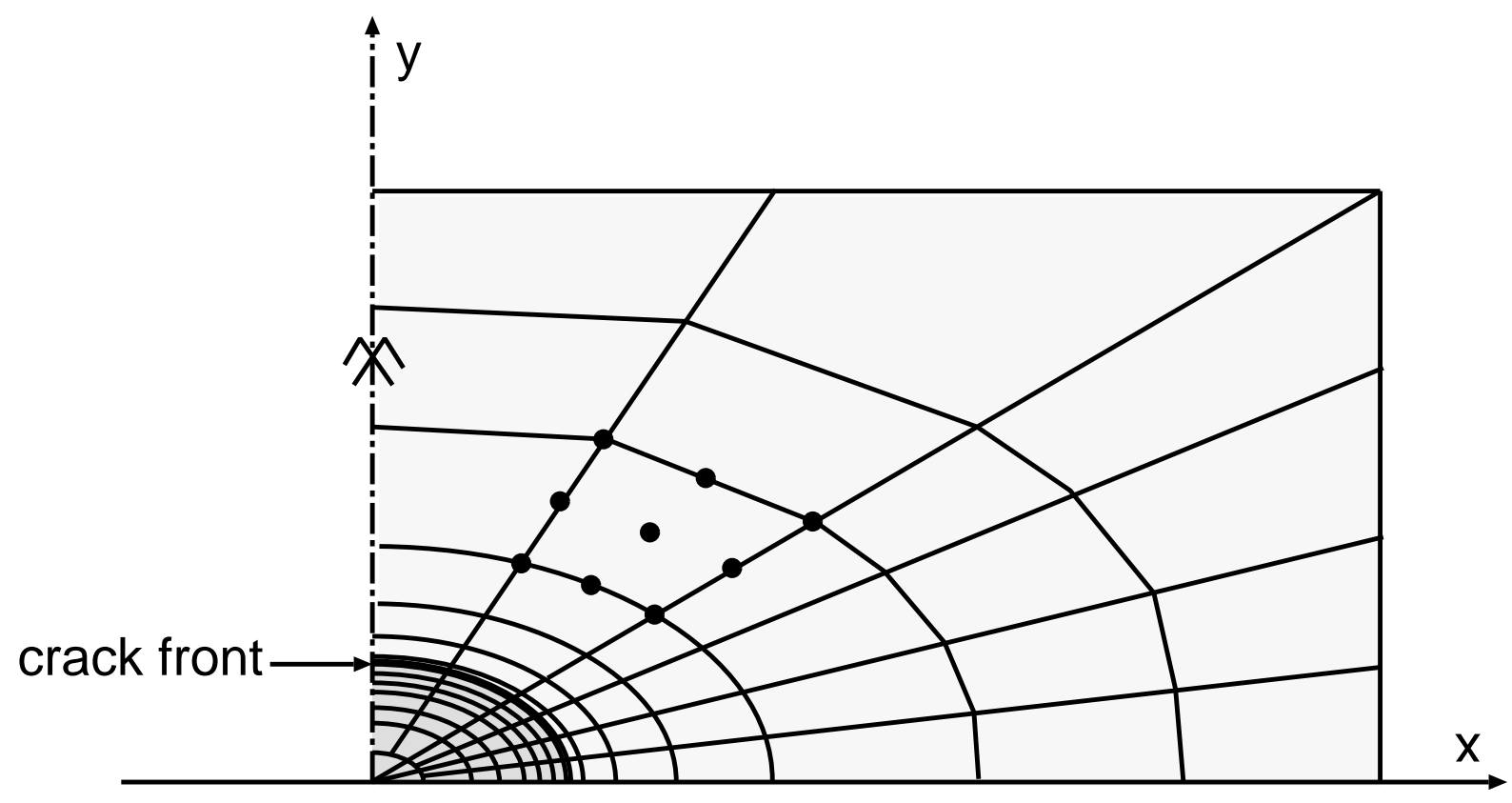

Figure 12: Example 2: Boundary element subdivision of crack plane (mesh $\mathrm{M} 2, b=0.6 a$ ) 


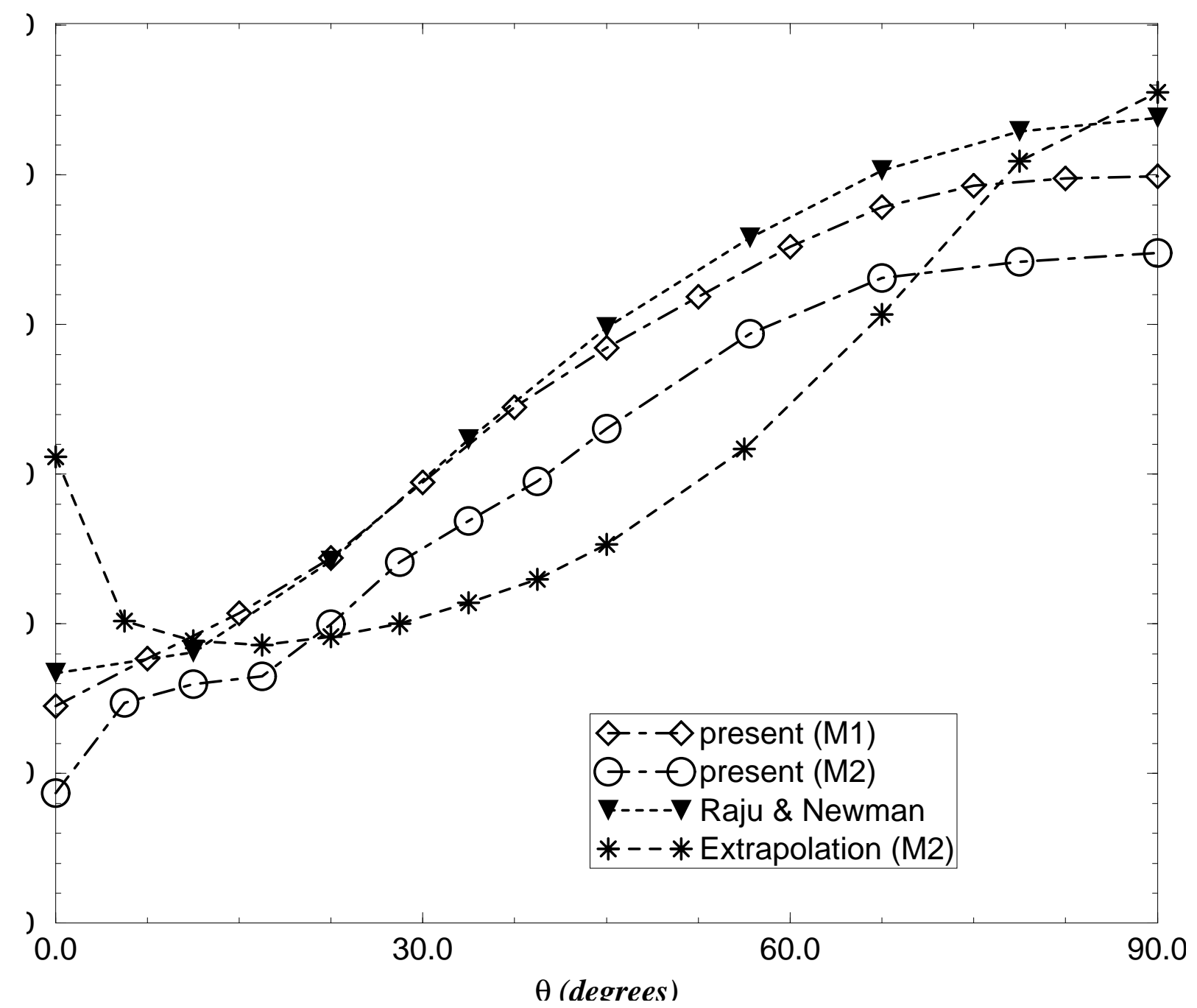

Figure 13: Example 2: comparison between results (shallow semielliptical surface crack, $b=0.4 a$ ) 


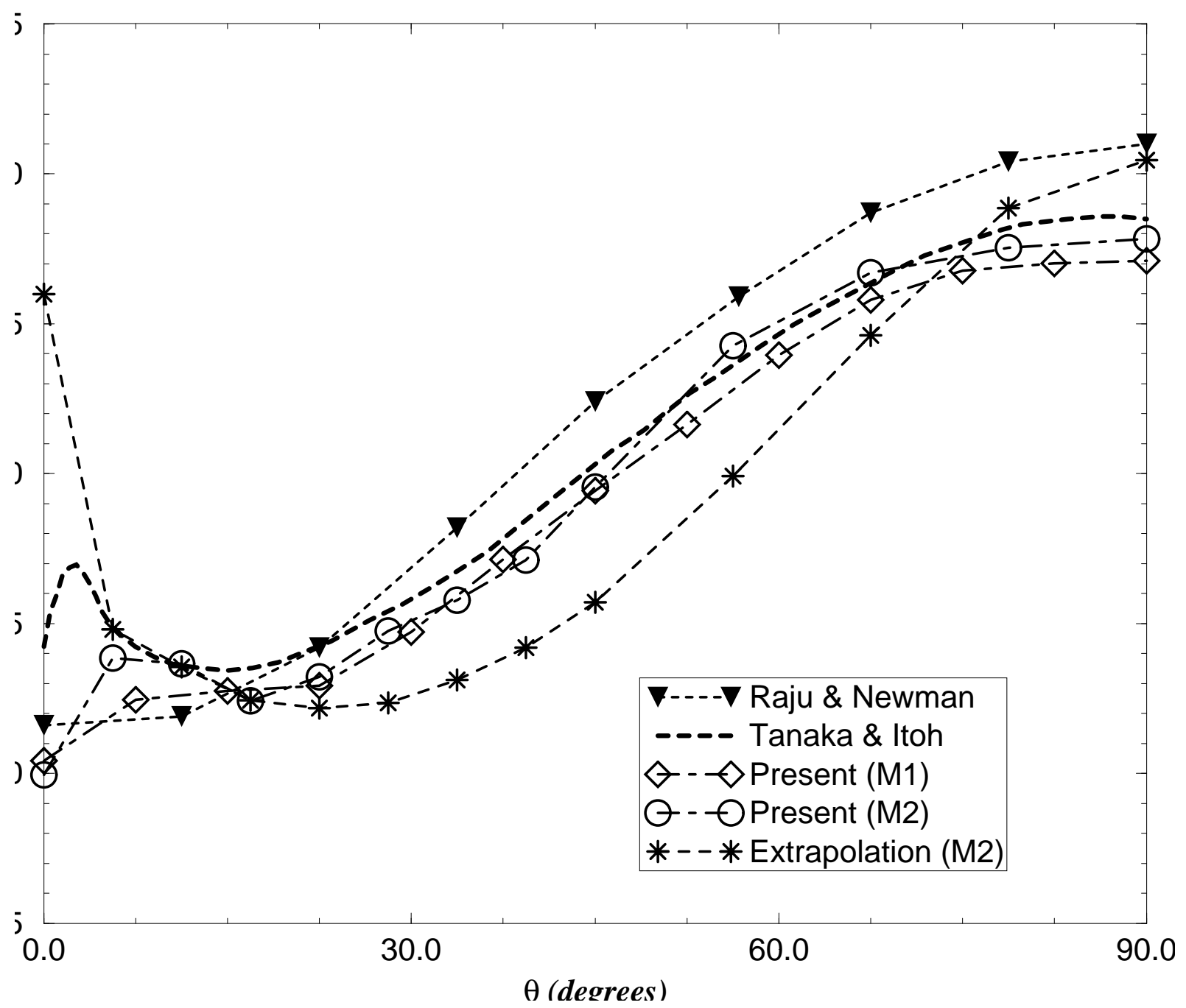

Figure 14: Example 2: comparison between results (shallow semielliptical surface crack, $b=0.6 a)$ 


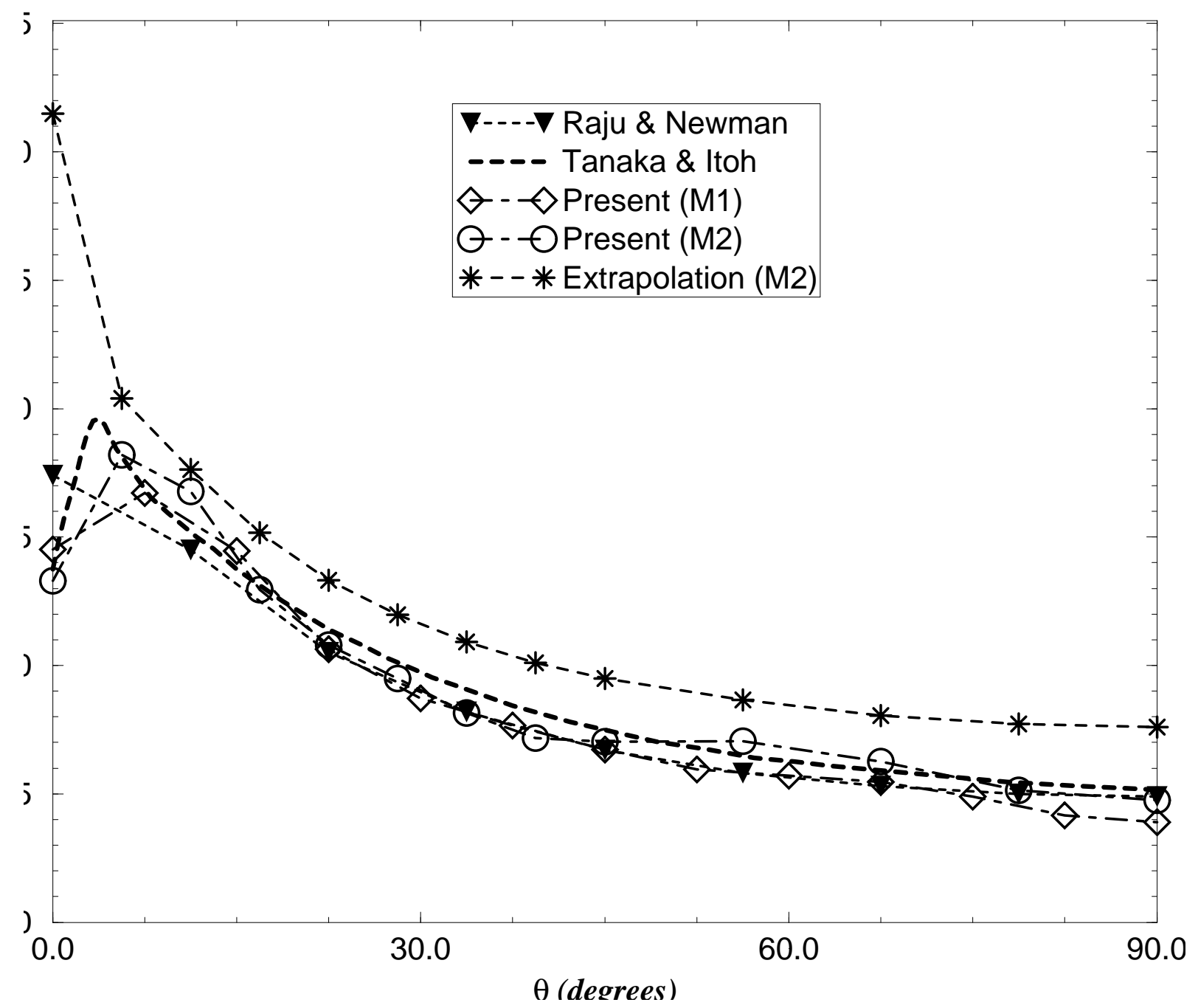

Figure 15: Example 2: comparison between results (semicircular surface crack, $b=a)$ 


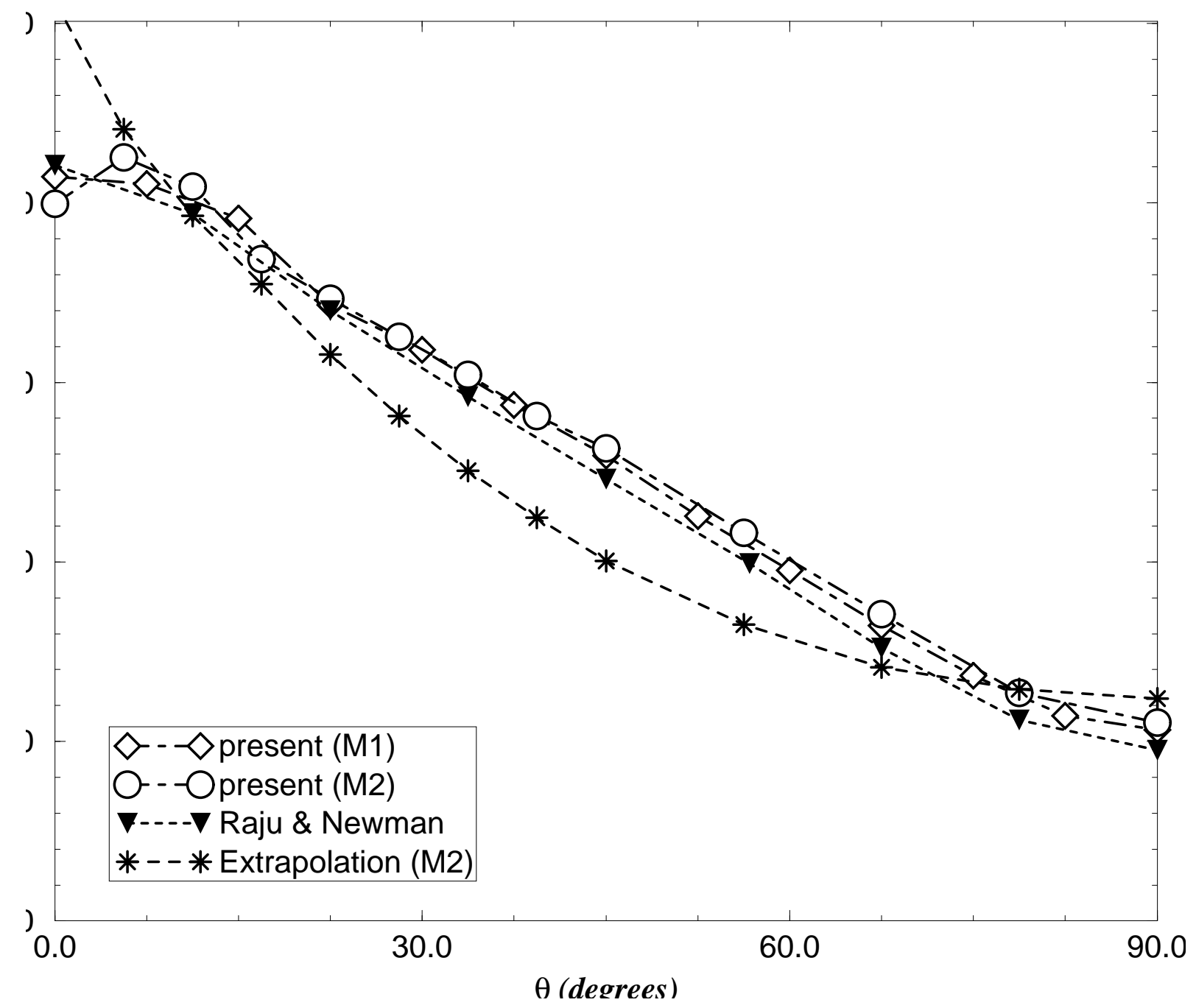

Figure 16: Example 2: comparison between results (deep semielliptical surface crack, $b=2 a)$ 